\title{
Meridional energy transport extremes and the general circulation of NH mid-latitudes: dominant weather regimes and preferred zonal wavenumbers
}

\author{
Valerio Lembo ${ }^{1}$, Federico Fabiano ${ }^{1}$, Vera Melinda Galfi ${ }^{2}$, Rune Graversen ${ }^{3,4}$, Valerio Lucarini ${ }^{5}$, and \\ Gabriele Messori ${ }^{2,6}$ \\ ${ }^{1}$ CNR-ISAC, Bologna, Italy \\ ${ }^{2}$ Department of Earth Sciences and Centre of Natural Hazards and Disaster Science (CNDS), Uppsala University, Uppsala, \\ Sweden \\ ${ }^{3}$ Department of Physics and Technology, UiT - The Arctic University of Norway, Troms $\varnothing$, Norway \\ ${ }^{4}$ Norwegian Meteorological Institute, Troms $\varnothing$, Norway \\ ${ }^{5}$ Department of Mathematics and Statistics and Centre for the Mathematics of Planet Earth, University of Reading, Reading, \\ UK \\ ${ }^{6}$ Department of Meteorology and Bolin Centre for Climate Research, Stockholm University, Stockholm, Sweden \\ Correspondence: Valerio Lembo (v.lembo@isac.cnr.it)
}

\begin{abstract}
The extratropical meridional energy transport in the atmosphere is fundamentally intermittent in nature, having extremes large enough to affect the net seasonal transport. Here, we investigate how these extreme transports are associated with the dynamics of the atmosphere at multiple scales, from planetary to synoptic. We use ERA5 reanalysis data to perform a wavenumber decomposition of meridional energy transport in the Northern Hemisphere mid-latitudes during winter and summer. We then relate extreme transport events to atmospheric circulation anomalies and dominant weather regimes, identified by clustering $500 \mathrm{hPa}$ geopotential height fields. In general, planetary-scale waves determine the strength and meridional position of the synoptic-scale baroclinic activity with their phase and amplitude, but important differences emerge between seasons. During winter, large wavenumbers $(k=2-3)$ are key drivers of the meridional energy transport extremes, and planetary and synoptic-scale transport extremes virtually never co-occur. In summer, extremes are associated with higher wavenumbers $(k=4-6)$, identified as synoptic-scale motions. We link these waves and the transport extremes to recent results on exceptionally strong and persistent co-occurring summertime heat waves across the Northern Hemisphere mid-latitudes. We show that these events are typical, in terms of dominant regime patterns associated with extremely strong meridional energy transports.
\end{abstract}




\section{Introduction}

The latitudinal gradient in incoming net solar radiation is the main trigger of atmospheric and oceanic dynamics (Peixoto and Oort, 1992). Indeed, the resulting energy imbalance induces an atmospheric circulation that causes divergence of moist-static energy at low latitudes and convergence at high latitudes.

In the low latitudes, a key role is played by the zonally averaged circulation, dominated by the Hadley circulation, and the energy transport embedded in the mass streamfunction (Holton and Hakim, 2012). In the mid-latitudes, eddies are the most significant contributors to the overall transport. Conventionally, the eddy contribution is partitioned into a transient and a stationary component, with the former identified as the instantaneous deviation from long-term climatology, and the latter as the climatological departure from the zonal mean transport (e.g. Starr and White (1954); Lorenz (1967)). Energy transports associated with transient eddies have been found to exhibit a large variability, with a number of sporadic extreme events exceeding the mean values by a few orders of magnitude (Swanson and Pierrehumbert, 1997; Messori and Czaja, 2013, 2015). This emphasizes the non-linear nature of baroclinic activity and its interactions at different wave-scales and with the zonalmean circulation (cfr. Kaspi and Schneider (2013); Messori and Czaja (2014); Novak et al. (2015)).

The conventional transient-stationary decomposition, separating spatio-temporal averages and anomalies, mixes different spatio-temporal scales and does not allow to identify how specific features of the multiscale atmospheric dynamics in the midlatitudes are linked to meridional energy transports. An alternative approach uses a Fourier decomposition of eddy modes, to isolate the contribution of different zonal wavenumbers to the overall transport (Graversen and Burtu, 2016). It has recently been extended, by means of a wavelet decomposition, to the investigation of regional features, such as extratropical cyclones (Heiskanen et al., 2020). Fourier decomposition has enabled to separate synoptic and planetary-scale contributions, and their respective roles in engendering extreme meridional energy transports (Lembo et al., 2019). While synoptic-scale eddies always allow for baroclinic conversion, and thus systematically contribute to a poleward transports, planetary-scale eddies can occasionally oppose the total transport. These results point towards the importance of modes of low-frequency variability in modulating the energy transport by eddies (Messori and Czaja, 2015; Messori et al., 2017) and the emergence of preferred weather regimes in the occurrence of intermittent meridional energy transport extreme events (cfr. Nie et al. (2008); Ruggieri et al. (2020)). In a quasi-geostrophic context, the conversion of available potential energy into kinetic energy (Lorenz, 1955) supports interpreting the energy transfers in terms of modes of atmospheric variability, although the limits of predictability often challenge our understanding of low frequency variability and weather patterns associated with it. Particularly, blocking events in mid-latitudes have proven to be very complex aspects of the circulation, so that understanding the mechanisms behind them (Woollings et al., 2018; Lucarini and Gritsun, 2020) and reproducing them in state-of-the-art models (Davini and D'Andrea, 2016; Schiemann et al., 2020) is still an open issue. Through recurrent and persistent blockings, low frequency variability manifests itself, especially in summer, in terms of heat waves (e.g. the 2010 summer event over Russia, Dole et al. (2011)), whose nature of intermittent and extreme events has been recently studied with different methodologies, such as rare events algorithms (Ragone and Bouchet, 2020, 2021) and large deviation theory (Gálfi et al., 2019; Galfi and Lucarini, 2021; Gálfi et al., 2021). 
Here, we leverage the Fourier decomposition approach of Graversen and Burtu (2016) to analyse the circulation anomalies and weather regimes associated with meridional energy transport extremes. We select the extremes with a rigorous methodology based on Extreme Value Theory (EVT) (Gnedenko, 1943). We then link the meridional structure of the wavenumberdecomposed extreme distribution to the emergence of anomalous weather regimes.

The paper is organised as follows: in Sect. 2 we describe the data, the methodology for extreme events selection and for the clustering of weather regimes. In Sect. 3a distributions of meridional energy transports, their wavenumber decomposition and their extremes as a function of latitude are described, while in Sect. $3 \mathrm{~b}$ clustering of geopotential heights is related to composites of extreme events and dominant wavenumbers. In Sect. 4 an interpretation of these results is given, in light of composite mean anomaly patterns of geopotential height fields associated with extreme transports, while Sect. 5 summarises the findings and outlines limitations and future perspectives.

\section{Methodology}

\subsection{Data}

Data for meridional energy transport and atmospheric circulation analyses are obtained from the ERA5 Reanalysis (Hersbach et al., 2020), retrieved from the Copernicus Data Storage (CDS). The data used covers December-January-February (DJF) and June-July-August(JJA) from 1979 to 2012, with a 6-hourly temporal resolution, a horizontal spatial resolution of $1^{\circ} \times 1^{\circ}$ and 137 vertical levels. We deem the 6-hourly sufficient to encompass the synoptic and larger modes of variability, as evidenced in recent analyses (Lembo et al., 2019). The choice of seasons is aimed at avoiding conflating regimes that are representative of the warm and cold seasons, as may happen in spring and autumn. The analysis is focused on the mid-latitudinal Northern Hemisphere channel, i.e. latitudes in the $30^{\circ} \mathrm{N}-60^{\circ} \mathrm{N}$ range. For the computation of moist static energy, specific humidity (q), air temperature (T), and geopotential height (z) are considered. For the retrieval of the weather regimes and the $k$-means clustering, daily geopotential height at $500 \mathrm{hPa}(\mathrm{z} 500)$ is used.

\subsection{Methods}

\subsubsection{Meridional energy transports and wavenumber decomposition}

The instantaneous meridional energy transport (or, more correctly, the enthalpy transport, cfr. Ambaum (2010); Lucarini and Ragone (2011)) is obtained as the scalar product of the meridional velocity $v$ and the total energy $E$, including the kinetic energy and the moist static energy $H$, defined as:

$H=L_{v} q+c_{p} T+g z$

where $q$ is the specific humidity, $T$ is the air temperature, $z$ is the geopotential height, $L_{v}, c_{p}$ and $g$ are the latent heat of vaporization, the specific heat at constant pressure, and the gravity acceleration, respectively. The transport of $E$ across a given 
latitude is computed with the vertical and zonal integration:

$\oint \int_{p_{s}}^{0} v E \frac{d p}{g} d x=\oint \int_{p_{s}}^{0} v\left(H+\frac{1}{2} \mathbf{V}^{2}\right) \frac{d p}{g} d x$

where $p_{s}$ is the surface pressure and $\mathbf{V}$ is the horizontal velocity vector. The sign convention is that transport is positive when northward directed, and negative when southward directed. The wavenumber decomposition is extensively discussed in Graversen and Burtu (2016), and in Lembo et al. (2019). We briefly summarize it here, recalling that the meridional energy transport as a function of zonal wavenumber $k$ can be written as:

$$
\hat{\mathcal{F}}_{0}(\phi)=D \int_{p_{s}}^{0} \frac{1}{4} a_{0}^{v} a_{0}^{E} \frac{d p}{g} \quad k=0
$$

$\hat{\mathcal{F}}_{k}(\phi)=D \int_{p_{s}}^{0} \frac{1}{2}\left(a_{k}^{v} a_{k}^{E}+b_{k}^{v} b_{k}^{E}\right) \frac{d p}{g} \quad k=1, \ldots, N$

85

$$
\begin{aligned}
& a_{k}^{\Psi}(t, \phi)=\frac{2}{D} \int \Psi(t, \phi, \lambda) \cos \left(\frac{k 2 \pi \lambda}{d}\right) d \lambda \\
& b_{k}^{\Psi}(t, \phi)=\frac{2}{D} \int \Psi(t, \phi, \lambda) \sin \left(\frac{k 2 \pi \lambda}{d}\right) d \lambda
\end{aligned}
$$

where $\Psi$ is either $v$ or $E, D=2 \pi R \cos (\phi)$ with $R$ being the Earth's radius, $k$ being the zonal wavenumber, and $t, \phi, \lambda$ the time, latitude and longitude, respectively. The highest zonal wavenumber $N$ is set to 20 , given that the spatial and temporal resolution of the ERA5 outputs poses a constraint to the modes of variability that can be resolved, and since it has been shown that the transport associated with wave number 0 to 20 constitute almost entirely the total transport (Graversen and Burtu, 2016). For sake of conciseness, we group wavenumbers into: $k=0$, reflecting zonal mean transports, $k=1-5$ for planetary-scale transports, $k=6-10$ for synoptic-scale transports and $k=11-20$ as mesoscale and lower spatial scale transports. The meridional circulation includes a zonal mean mass transport in opposite direction at different altitudes. However, at small temporal scales a vertical mean mass transport across latitudes may be encountered, which may transport a large amount of energy. This latter component of the enthalpy transport has to be subtracted from wave zero transport, in order to obtain the energy transport by the mean meridional circulation (Liang et al., 2017), which is done following Lembo et al. (2019). The instantaneous zonal mean and vertical mean meridional-circulation energy transport component is thus written as:

$$
\left\{[v]^{\dagger}[E]^{\dagger}\right\}=\{[v][E]\}-\{[v]\}\{[E]\}
$$

with \{\} denoting the vertical mean, [ ] the zonal mean and $\dagger$ the departure from the vertical mean. Note that the wave zero energy transport component is given by the first term on the r.h.s. of Eq. 7. 


\subsubsection{Selection of the extreme events}

We adopt in this work a rigorous methodology for the detection of meridional energy transport extremes based on the "peak over threshold" approach of Extreme Value Theory (EVT) (Balkema and Haan, 1974; Pickands, 1975). The main advantage of this methodology is that the properties of the data themselves determine the threshold used to define extreme events instead of arbitrary threshold choices. Furthermore, the probability distribution of extreme values is given by EVT, thus no subjective decisions are needed considering the shape of this distribution. An overview of this approach is hereby drawn, based on Coles et al. (2001), and of the convergence algorithm also presented in Gálfi et al. (2017). Full theoretical and technical details can be found therein.

We first consider a series of independent identically distributed random variables $X_{1}, X_{2}, \ldots$ with common probability distribution $F(X), X$ denoting an arbitraty term in the series $X_{i}$. Under appropriate conditions (discussed generally below, for a comprehensive and rigorous description see Leadbetter (1974); Leadbetter et al. (1989); Coles et al. (2001)), the threshold exceedances $y$ of a large enough threshold $u, y=X-u$ with $X>u$, are asymptotically distributed according to the Generalized Pareto Distribution (GPD) family as:

$$
\begin{array}{ccc}
H(y)=1-\left(1+\frac{\xi y}{\sigma}\right)^{\frac{1}{\xi}} & \text { for } & \xi \neq 0 \\
H(y)=1-\exp ^{-\frac{y}{\sigma}} & \text { for } & \xi=0
\end{array}
$$

with $1+\xi y / \sigma>0$ for $\xi \neq 0, y>0$, and $\sigma>0$. The shape parameter $\xi$ determines the shape of the distribution and the tail behavior. If $\xi=0$ the tail of the distribution decays exponentially, if $\xi>0$ the tail decays polinomially, while, if $\xi<0$, the distribution is bounded.

In order to apply EVT to meridional energy transport data, one has to make sure that the conditions of independence and homogeneity are fulfilled, and thus the data can be modelled based on a stationary stochastic process (Leadbetter, 1974). While both conditions are, strictly speaking, unrealistic in case of geophysical data, the problem can be approached from a practical perspective. First, we detect linear long-term trends for the 1979-2012 period, and we test their significance according to a Mann-Kendall test at the 95\% significance level (Mann, 1945; Forthofer and Lehnen, 1981), then we remove trends only at those latitudes where they are found to be significant, i.e., for DJF, north of $37.5^{\circ} \mathrm{N}$, for JJA in the $40^{\circ} \mathrm{N}-47.5^{\circ} \mathrm{N}$ and $54.5^{\circ}$ $\mathrm{N}-58.5^{\circ} \mathrm{N}$ latitudinal bands. By analysing summer and winter separately, we eliminate another source of dependence and heterogeneity due to the seasonal cycle. Furthermore, we verify for every latitude the decay of the auto-correlation function. Despite the fact that several consecutive values in our times series are correlated, the chaotic nature of atmospheric motions allows that two values being far away in time from each other are nearly independent, meaning that the auto-correlation function decays to 0 at a finite time lag. Looking at the auto-correlation functions, we find that JJA (DJF) daily data require the removal of the climatological JJA (DJF) season at every latitude (south of $45^{\circ} \mathrm{N}$ ). Once this is achieved, the GPD parameters may be computed. Even in the absence of long-range correlations, however, one may encounter clusters of data exceeding the threshold consecutively in time. Those are clearly correlated and would lead to biased GPD parameters ${ }^{1}$ as a result of a slower

\footnotetext{
${ }^{1}$ The asymptotic GPD shape parameter is not affected by the existence of clusters, its finite-size estimates however are biased due to the slow convergence.
} 
convergence to the limiting distribution (Coles et al., 2001). To avoid this, we decluster, where necessary, the time series before estimating the parameters by using the "intervals" declustering method (Ferro and Segers, 2003) based on the "extremal index" defined in Gálfi et al. (2017).

We then test the applicability of the theory by looking at the convergence of the selected extremes to the limiting GPD distribution. This is done by plotting the estimated shape parameter $\xi(u)$ as a function of an increasing threshold $u$. The lowest threshold $u^{*}$ at which the shape parameter "plateaus", i.e. does not change with further threshold increases, $\xi\left(u^{*}\right) \approx \xi\left(u>u^{*}\right)$, provides the optimal parameter estimate. This estimate has a lower uncertainty than for $u>u^{*}$, and at the same time provides an estimated distribution which is equivalent to the limiting GPD considering the estimation uncertainty given by the confidence intervals. In order to achieve a latitude-independent definition of extremes, we express the threshold in terms of fraction of data points above the threshold.

This threshold selection procedure applies in case of extremes located in the right tail of the transport's probability density function (pdf), hereafter referred to as "poleward" extreme energy transports. Similarly, we refer to extremes located in the left tail of the pdf as "equatorward" extreme energy transports. These extremes are weaker than the median transports, and sometimes slightly negative. The procedure for equatorward extremes is the same as described above for poleward extremes. Hence, we search for a stable shape parameter as function of a decreasing threshold, equivalent to a decreasing fraction of data points below the threshold.

For illustrative purposes, Figure 1 shows the behavior of the shape parameter $\xi$ as a function of the threshold $u$ at different latitudes. We notice that the shape parameter is almost everywhere negative, evidencing that the extreme value distribution is bounded. In other words, Figure 1 provides a graphical explanation of the convergence methodology, justifying the following choice of the thresholds:

- DJF, equatorward: $10 \%$ of data below the threshold (10\% percentile);

- DJF, poleward: $14 \%$ above the threshold (86\% percentile);

- JJA, equatorward: $14 \%$ below the threshold (14\% percentile);

- JJA, poleward: 7\% above the threshold (93\% percentile);

We notice that, while $\xi$ is systematically negative for this choice of the thresholds, $\sigma$ is positive and slightly increasing with latitude, denoting an increasingly large spread of extremal values (Fig. 1a, b).

\subsubsection{Weather regimes}

The Weather Regimes (WRs) are computed from the daily geopotential height at $500 \mathrm{hPa}$ (z500), using the WRtool Python package (Fabiano et al., 2021). We summarise the methodology here; for more details and discussion the reader is referred to the Methods section in Fabiano et al. (2020). We focus on latitudes between $30^{\circ}$ and $90^{\circ} \mathrm{N}$ and three different longitudinal sectors: the Euro-Atlantic (EAT, from $80^{\circ} \mathrm{W}$ to $40^{\circ} \mathrm{E}$ ), the Pacific-North American (PAC, from $140^{\circ} \mathrm{E}$ to $80^{\circ} \mathrm{W}$ ) and the whole Northern Hemisphere mid-latitudes (NH). 
The original data were first interpolated to $2.5^{\circ} \times 2.5^{\circ}$ horizontal resolution; since the WR diagnostic aims at capturing largescale configurations, this step does not alter the results of the analysis. Before computation, the daily mean seasonal cycle smoothed with a 20-day running mean - is removed from the data to obtain daily anomalies.

To retain only the large-scale component and reduce dimensionality, an Empirical Orthogonal Function (EOF) decomposition is performed, separately for each considered longitudinal sector. The minimum number of EOFs that explain at least $90 \%$ of the total variance is retained: this corresponds to 16, 19 and 41 EOFs during DJF for the EAT, PAC and NH, respectively; for JJA 22, 25 and 57, respectively. The WRs are then computed by applying a $K$-means clustering algorithm to the selected Principal Components (PCs). For all sectors and seasons, we set the number of regimes to 4 . This choice is widely documented in literature for the EAT and PAC winter circulation (Michelangeli et al., 1995; Cassou, 2008; Weisheimer et al., 2014; Hannachi et al., 2017; Straus et al., 2017), although different numbers of clusters have been used in other studies (e.g. Pasquier et al., 2019; Strommen et al., 2019; Hochman et al., 2021). Each day is assigned to one of the regimes and a set of 4 regime centroids is obtained. The regime pattern is defined as the composite of all anomalies assigned to a certain regime.

Figure 2 shows the pattern of the weather regimes computed for each region (EAT, PAC, NH) and season (DJF, JJA) during 1979-2013, following the procedure described above. DJF patterns (left column) resemble the conventional DJF weather regimes in the literature. These are, for EAT: the North Atlantic Oscillation (NAO) positive phase (hereafter NAO+), the Scandinavian blocking (SC), the Atlantic ridge (AR), the NAO negative phase (NAO-) (Cassou, 2008; Dawson et al., 2012); for PAC: the Pacific trough (PT), the positive and negative phases of the Pacific-North American pattern (PNA+, PNA-), the Bering ridge (BR) (Jung et al., 2005; Weisheimer et al., 2014; Straus et al., 2017); for NH: the "Cold-Ocean-Warm-Land" pattern (COWL), the PNA-, the Aleutinian ridge (ALR), the Arctic Oscillation negative pattern (AO) (cfr. Kimoto and Ghil (1993); Corti et al. (1999)).

We refer to JJA regimes (right column) as $x C n$, with "x" being EAT, PAC or $\mathrm{NH}$, and $n=1, \ldots, 4$ the index denoting the cluster. Thus, for the EAT domain: EATC1 features a zonally spread blocking extending from the Eastern Atlantic to Scandinavia, $E A T C 2$ closely resembles the NAO+ pattern, EATC3 can be referred to as a Greenland blocking, while EATC4 features a dipole structure, with an Eastern Atlantic low and a Scandinavian high (cfr. Guemas et al. (2010); Yiou et al. (2011)). Concerning PAC and NH, all regimes feature anomalies that are much weaker than DJF regimes, given that the circulation as a whole is weaker in the JJA season. $P A C C 1$ features a weak blocking south-west of Alaska, while $P A C C 2$ is somehow similar to the BR pattern, although much weaker. $P A C C 3$ and $P A C C 4$ appear to mirror each other. $N H C 1$ exhibits a Siberian high, while $\mathrm{NHC} 2$ features a number of highs surrounding the North Pole; $\mathrm{NHC} 3$ features a Greenland blocking and $\mathrm{NHC4}$ closely resembles $E A T C 1$.

For each cluster, the absolute frequency is computed within the population of all events, then the ratio of absolute frequency in the population of extreme events to the absolute frequency in the overall population is obtained, for each tail of the distribution and in each season. Specifically, the relative variations in absolute frequencies are retrieved as $\Delta f_{x}^{\prime}=\frac{f_{x}^{\prime}}{f_{x}}-1$, where $f_{x}$ is the absolute frequency of occurrence of the cluster $x$ for the overall population, and $f_{x}^{\prime}$ is the frequency of occurrence of extreme events in cluster $x$ relative to the total extreme population. Hence a positive $\Delta f_{x}^{\prime}$ indicates that cluster $x$ include a higher fraction of extreme events compared to its fraction of all events, i.e. cluster $x$ is more favourable for extreme events. In 
order to assess whether the variation in absolute frequencies is significant, a bootstrapping is performed, through 300 random resamplings of the original population with the same number of events as the number of extreme events in the respective tail. The hypothesis test is performed at the one-sided $95 \%$ significance level.

In order to link the weather regimes characterisation of extremal transports to the wavenumber decomposition illustrated in Sect. 3.1, we focus on the zonal wavenumber effecting the largest share of the meridional energy transport anomaly. First, at each latitudinal band, for each assigned weather regime, in each season and for each tail of the distribution, we compute the contribution of each individual wavenumber to the meridional energy transport anomaly wrt. the average across the extreme events in the selected subset. Then, the dominant wavenumber for the specific event is assigned, as the one whose contribution to the transport is largest. Finally, the average of the dominant wavenumbers across the subset of extreme events assigned to the specific regime and occurring at the selected latitudinal band is computed.

\section{Results}

\subsection{The PDFs of meridional energy transports and their wavenumber decomposition}

We start our analysis by looking at the PDFs of the total and wavenumber-decomposed meridional energy transports and their extremes.

The normalized PDFs (filled contour plots) of the total zonally averaged meridional energy transport are computed for each latitude in the $30^{\circ}-60^{\circ}$ latitudinal channel, as shown in the top panels of Figures 3 and 4 (for DJF and JJA, respectively). In DJF, the PDF peaks at about $42.5^{\circ} \mathrm{N}$, with a value of $6.5 \times 10^{15} \mathrm{~W}$, and then steeply decays towards the high latitudes. In JJA, the PDF shows a weaker variability across latitudes, with a mean/median value oscillating between 2.0 and $2.5 \times 10^{15} \mathrm{~W}$, and a moderate decrease towards the high latitudes. PDFs in DJF are mildly positive-skewed (with higher skewness at the edges of the midlatitudinal channel, amounting to 0.96-1.09, and lower skewness in the midlatitudes, about 0.85). The low latitudes in JJA clearly reflect a positively skewed distribution, whereas the PDF is more symmetric in the high latitudes data (with skewness ranging between 1.28 in the low latitudes and 0.96 in the high latitudes).

The tails of the distributions, encompassing the extreme events selected according to the methodology described in Sect. 2.2.2, are skewed towards either equatorward or poleward extreme transports in both seasons. Equatorward extremes attain smaller or even negative values at higher latitudes, where the mean transport is weaker, which emphasises the importance of such extreme events for setting the seasonal energy transport at high latitudes. The opposite occurs for the poleward extremes, with the largest extremes being stronger for those latitudes where the mean transport is stronger. This is in agreement with Lembo et al. (2019), who found that both poleward and equatorward extremes are related to the mean strength of the transport (cfr. their Figure 1d-g).

We next decompose the transport into its wavenumber components (panels c)-f) in Figures 3 and 4). Not surprisingly, in both seasons the most significant contribution to the overall transport comes from the planetary-scale and synoptic-scale contributions. The equatorward and poleward extremes largely overlap in the zonal mean component, and both attain negative values. Very different patterns emerge at the planetary and synoptic scales. During DJF and at both these scales, the magnitude 
of poleward extremes follows that of the median transport for the respective component, and attains large values when the median component is at its maximum. Equatorward extremes, on the other hand, only partly follow the median curve and attain near-zero values at almost all latitudes, especially regarding the synoptic contribution. During JJA, a significant share of planetary-scale equatorward extremes feature net negative transport in the middle latitudes, where the contribution to the transport is almost vanishing on average. This is consistent with planetary waves being able to act passively, transporting energy counter-gradient, and thus with no conversion of available potential energy into kinetic energy, as discussed in Baggett and Lee (2015); Lembo et al. (2019). Synoptic-scale waves, instead, almost everywhere share the positive sign and the meridional structure of the mean transport, although as in DJF, the most extreme events during JJA also attain near-zero values at almost all latitudes.

\subsection{Weather regimes and zonal wavenumbers associated to extreme events}

We now shift our attention to the detection of weather regimes in the population of extreme events, in order to investigate the relation between extreme transports and recurrent patterns of the large-scale circulation.

As discussed in Appendix B, conditioning on different extreme transport events yields different average values of the PCs of the leading EOFs, suggesting that the population of extreme events might exhibit a preferred development of specific weather regimes. Following from this hypothesis, we compute $\Delta f_{x}^{\prime}$ in the population of extreme events (as described in Sect. 2.2.3), grouping extremes by 2-degrees wide latitudinal bins. Figures 5-6 display $\Delta f_{x}^{\prime}$ for each of the chosen regimes in the EAT, PAC and $\mathrm{NH}$ regions, for poleward (left) and equatorward (right) extreme events in DJF and JJA, respectively.

DJF poleward extremes are characterized by anomalously negative z500 anomalies in the lower mid-latitudes and highlatitudinal blockings, especially over the Northern Atlantic, as denoted by the sharp increase in the frequency of NAO-, AO and PT regimes. Consistently, a significant reduction in the NAO+, BR and ALR modes is found. In JJA, NHC4/EATC2, characterised by a pattern similar to an Eastern Atlantic-Scandinavian blocking, is increasingly likely. EATC4 and PACC4, denoted by negative anomalies in the eastern boundaries of the Atlantic and Pacific oceans, are rarer, as well as NHC3, featuring a weak Pacific ridge and a Greenland blocking. In general, we observe that changes in absolute frequencies are coherent across neighbouring latitudes, consistently with what is observed for the energy transport extremes, often occurring concomitantly at several latitudes (compare to Fig. 1d-g in Lembo et al. (2019)).

DJF equatorward extremes largely mirror what is described for poleward extremes: NAO-, PT and AO modes become generally less frequent, whereas NAO+, PNA-, ALR and, to some extent, BR modes are more frequent. In other words, a more zonal North Atlantic regime and stronger Pacific blocking are associated with weaker meridional transports. This is in line with the opposite signs of PC fractional changes described in Appendix B. Looking at JJA, it clearly emerges that the PACC4 pattern becomes more frequent, while $P A C C 3$ and $N H C 3$ (especially for extremes at high latitudes) are rarer. Remarkably, $E A T C 3$ and $E A T C 1$ become increasingly likely for extremes at low and mid-latitudes, respectively, EATC2 for extremes at higher latitudes. This suggests a weakening and a northward shift of the centres of baroclinic activity, as the latitude at which the equatorward extremes are selected increases. 
We then focus our attention to the dominant wavenumber, as a function of the region and of the cluster to which extreme events are attributed. We remark that the spectrum of the meridional energy transports is now different from the one considered in Figures 3 and 4, as we focus on the wavenumber decomposition anomalies relative to their climatological contributions, rather than on their absolute values. For this reason, we limit our range of wavenumbers to $0-7$, as no higher wavenumber appears to be dominant in any of the considered cases.

No matter in which region the clustering is focused, DJF extreme events are associated with wavenumbers between 2 and 4 (Figure 7). In JJA (Figure 8), the situation is more diversified, with wavenumbers in the range of $k=[2, \ldots, 6]$ and a clearer dependence on the circulation cluster. The dominance of higher zonal wavenumbers is consistent with the finding that PC fractional changes in JJA occur mainly for higher order EOFs, as described in Appendix B. Poleward extremes generally peak at higher wavenumbers than equatorward extremes. Focusing on $E A T C 2$ and $N H C 4$, we also find that synoptic scales $(k=5$ and $k=6$ ) are co-located with latitudes featuring the largest frequency change (cfr. Figure 6). Interestingly, to some extent the same happens for EATC4, PACC4 and $N H C 3$, where the latitudes featuring the largest frequency reductions are also characterised by relatively higher wavenumbers (compared to extremes in other clusters and in the same cluster but different latitudes), again $k=5$ and $k=6$. Dominant wavenumbers for equatorward extremes are less sensitive to frequency variations: events associated with $P A C C 4$ and $E A T C 1$ frequency increases are associated with a dominant $k=4$ wavenumber. EATC2 features a $k=3$ dominant wavenumber in the high latitudes, whereas $E A T C 3$ is characterised by a $k=2$ wavenumber in the low latitudes.

\section{Discussion}

Composite mean z500 anomalies for the subset of extremes taken at different latitudes (Figures 9 and 10) support the message contained in the results above. Clearly, JJA and DJF differ in the fact that the higher zonal variability in the latter is related to a higher dominant zonal wavenumber. In both seasons, poleward extremes are characterised by a more marked meridional structure of the z500 anomalies compared to equatorward extremes, confirming that the zonality of the general circulation (and the dominant patterns denoting it) is related to the strength of the meridional energy exchanges.

Going into the details of the different seasons and tails of the distributions, we start from JJA equatorward extremes, given that they exhibit an a priori surprisingly large share of energy exchanges from the Pole toward the Equator. Lower latitudinal extremes are associated with widespread negative z500 anomalies, both on the main ocean basins and on land. For higher latitudinal extremes, a Euro-Scandinavian blocking pattern emerges, consistently with an increasing frequency of the EATC2 pattern, with negative z500 anomalies confined at even higher latitudes, and with a dominant $k=2-3$ wavenumber pattern. An interpretation of these features is provided in light of what described above (cfr. Figures 6 and 8). At lower latitudes (30-33 ${ }^{\circ}$ $\mathrm{N})$, the increased likelihood of the $P A C C 4$ regime is consistent with co-located negative anomalies, especially over the Pacific, and a dominant $k=4$ wave. Overall, this hints at the role of the planetary-scale contribution, that is particularly relevant when the total transport extreme is negative and the synoptic contribution is weak, especially at higher latitudes, as seen in Figure 4. Looking at the composites, we thus argue that, when the extreme transport is negative, the planetary-scale transport in the 
https://doi.org/10.5194/wcd-2021-85

Preprint. Discussion started: 4 January 2022

(c) Author(s) 2022. CC BY 4.0 License.

Weather and

Climate Dynamics

Discussions

L

subset of extreme equatorward transports in JJA, acts by confining baroclinic eddies towards the high latitudes, overwhelming the synoptic-scale transport.

JJA poleward extremes also find a relatively straightforward interpretation in the z500 composites. For extremes located in the 30-33 band, negative z500 anomalies are widespread (although with very different patterns compared to the equatorward extremes), while at higher latitudes several blocking patterns emerge in the channel. Overall, the regime frequency changes (in particular the reduced frequency of the EATC4, PACC4 and NHC3 regimes) are consistent with this pattern, with the midlatitudinal negative anomalies being replaced by coexisting high geopotential ridges. Consistently with Figure 8, evidencing that these patterns are dominated by wavenumbers $k=5-6$, Kornhuber et al. (2020) find that these modes of the general circulation are associated with the development of co-located heat waves in the NH Summer. We thus argue that heat waves may plausibly be related to the occurrence of extremely strong poleward meridional energy transports.

To heuristically test this hypothesis, we focus on poleward extreme transports in JJA for the year 2010, which was characterised by a number of concurrent heat waves developing in several regions of the $\mathrm{NH}$ mid-latitudinal channel, especially Central-Eastern North America and Russia (Dole et al., 2011). Figure 11 shows analogous results to those shown in Figures 6, 8 and 9, for JJA 2010 only. Extremes at the three chosen latitudinal bands are associated with a positive anomaly over Eastern Europe and Russia, particularly intense in the 45-47 latitudinal band. The composites are also consistent with an increased frequency of the $E A T C 2$ and $N H C 4$ patterns (Figure 11b), especially in the higher latitudinal bands. In addition to that, some frequency reductions, that are not as relevant in the rest of the extreme events population, are found for EATC1, PACC1 and $N H C 1$, pointing towards a reduced occurrence of a NAO+ regime and of the so-called "Aleutinian ridge". Kornhuber et al. (2020) interpreted the 2010 heatwave as a case study of concomitant heatwaves over several regions of the Northern Hemisphere, a consequence of quasi-resonant amplification of stationary Rossby waves (Kornhuber et al., 2017; Petoukhov et al., 2013). They found that $k=5--7$ were particularly favorable to the development of this pattern. Here, we show (Figure 11c) that more frequent regimes in the energy transport extremes population are associated with dominant wavenumbers ranging between $k=4$ and $k=6$. Overall, we thus argue that the 2010 event is "typical" of the more general circulation features associated with poleward meridional energy transport extremes in JJA, and that their occurrence is consistent with the explanation given by (Kornhuber et al., 2020) and the quasi-resonant amplification mechanism. The fact that the 2010 event captures a rare yet typically persistent fluctuation of the summer atmospheric fields in Eurasia has been discussed by Galfi and Lucarini (2021) using large deviation theory arguments. We also found (not shown) that the so-called "Dzud" event occurred in Winter 2010, characterized by intense and persistent cold outbreaks throughout parts of Asia, has been typical (in the sense described above) in terms of meridional energy transport extremes, and associated weather regimes.

Looking at DJF z500 composites (Figure 10), we note that the three latitudinal bands are characterised by a NAO- pattern, when poleward extremes are taken into account. In the Pacific-North American sector, a dipole structure, somewhat opposed to what found in JJA, emerges, with a strong trough in the Pacific and a ridge over the American continent at 45-47, moving westward and weakening at 57-60. The described pattern is consistent with what found in Figure 5, the NAO-, PT and AO weather regimes becoming more frequent for extremes at all latitudes. At the same time, the Pacific dipole structure is opposed at higher latitudes by negative anomalies, consistently with a reduction in high-latitudinal blockings over that region, as denoted 
by the decreased frequency of ALR and BR regimes. Composites are also consistent with $k=2-3$ being the range of dominant wavenumbers for the DJF poleward extremes, as seen in Figure 7, with a relatively weak dependence on the weather regime assigned to the extreme event under consideration. The pattern for DJF equatorward extremes is to a certain extent reversed wrt. poleward extremes, with a clear NAO+ footprint in the poleward half of the mid-latitudinal channel, and a ALR-like pattern for extremes located in the $45-47^{\circ}$ channel. This reflects the fact that the dominant $k=2--4$ wavenumbers do not change for the two classes of extremes, no matter the regime to what these are assigned (cfr. Figure 7). It is rather the phase and amplitude of ultra-long planetary-scale waves, carrying energy from the Equator towards the Pole for both poleward and equatorward meridional transport extremes (cfr. Figure 3c), that determine the strength and location of the overall baroclinic eddy activity.

In DJF, planetary and synoptic-scale transport extremes thus rarely co-occur (as already stressed in Lembo et al. (2019) and above in this work), and the planetary contribution to the extreme transport is less dependent on the median value of the transport than the synoptic contribution. In other words, in DJF the planetary-scale pattern, embedding higher-scale eddies, is dominant almost everywhere except at lower latitudes, and the synoptic scale contribution sharply weakens with the median transport as the latitude increases. Synoptic-scale eddies are thus modulated by the phase and strength of the underlying dominant planetary wave, as clearly suggested by the $500 \mathrm{hPa}$ geopotential anomalies. On the contrary, the strengths of the planetary- and synoptic-scale contributions are comparable in JJA. The pattern highlighted by the composite analysis is thus a blend of the two competing factors, with synoptic-scales becoming dominant in central latitudes (for poleward extremes) or the two contributions canceling each other almost symmetrically at most latitudes, confining baroclinic activity where the extreme transports occur (for equatorward extremes).

Summarizing, the composite analysis illustrates qualitatively that the extremes in the overall transport are the result of the interference of high and low wavenumbers (see also the discussion in Messori and Czaja (2014) and Messori et al. (2017) on this topic). Such interference occurs in different ways in the two seasons, with the planetary-scale waves generally determining the latitude and strength of the baroclinic activity, but relative role of the synoptic and planetary waves being very different at different latitudes. The minima and maxima of the planetary and synoptic-scale transports are in fact located at the two edges of the mid-latitudinal channel in DJF, and at the centre of the channel in JJA (cfr. Figures 3c-d and 4c-d).

\section{Summary and Conclusions}

In this work, we analysed the zonally averaged meridional energy transports in the mid-latitudinal NH channel for the DJF and JJA seasons in ERA5 Reanalysis. We decomposed the transports depending on their zonal wavenumbers, and isolated four contributions: zonal mean $(k=0)$, planetary scales $(k=1--5)$, synoptic scales $(k=6--10)$ and higher scales $(k=$ $11--20)$. We applied an EVT-based selection methodology to isolate extreme meridional energy transport events, labelled as "poleward", when the transport is stronger than average, "equatorward" when it is weaker. We adopted a clustering algorithm for the z500 anomalies associated with meridional energy transport extremes, and we looked for dominant patterns and preferred wavenumbers as a function of the latitudes at which the extremes are detected. We used this to support our interpretation of geopotential height composites of extreme transport events. 
We find that energy transport extremes emerge as a result of the growth of different wave types across the mid-latitudes, particularly synoptic and planetary scales depending on meridional location and season. Specifically, dominant patterns and preferred wavenumbers suggest that planetary scales determine the strength and meridional position of the synoptic-scale baroclinic activity with their phase and amplitude, exhibiting significant seasonal differences. In DJF, they modulate the synopticscale activity, which is generally stronger than the smaller scales, while in JJA they chiefly interfere with the latter being of similar magnitude, either constructively (poleward extremes) or destructively (equatorward extremes). Notably, equatorward extremes feature mainly negative-signed planetary-scale transports north of $42^{\circ} \mathrm{N}$.

Understanding meridional energy transport extremes is key to identify mechanisms through which diabatic heating and temperature gradients are balanced. We demonstrated that some preferred regimes favour extreme transports, and that they reflect to some extent preferential modes of variability related to specific zonal wavenumbers. This has clear implications for high latitude warming/cooling, as already stressed before regarding the influence of synoptic-scale eddy activity on Arctic weather (cfr. Ruggieri et al. (2020)) or dynamical patterns related to extreme near-surface temperatures over the Arctic (cfr. Messori et al. (2018); Papritz (2020)).

The emergence of dominant zonal wavenumbers associated with extreme meridional energy transports also has implications for teleconnections. Investigations based on large deviation theory have shown that persistent weather extremes are associated with very large scale atmospheric features Gálfi et al. (2019); Galfi and Lucarini (2021); Gálfi et al. (2021). We emphasized here the similarity between our results and the concurrent emergence of heat waves across the Northern Hemisphere associated with a persistent $k=5--7$ Rossby wave pattern, as found by Kornhuber et al. (2020). Our results emphasize that the modes related to energy transport extremes are hemispheric in scale, and suggest that regional features such as the NAO or PNA trigger co-variability of weather in remote regions Thompson and Wallace (1998); Branstator (2002). Analysing the aggregated effect of wave packets on meridional energy transports in association with weather regimes provides a thermodynamic background to the concept of quasi-resonant amplification Petoukhov et al. (2013); Coumou et al. (2014); Kornhuber et al. (2017) for the development of weather extremes, potentially linking local events to anomalies in the general circulation.

Here, we estimated the extremal index with the aim to decluster the energy transport time series in order to accelerate the convergence of extreme value statistics. The extremal index itself, however, contains valuable information related to the persistence of extreme states, as it is the inverse of the mean cluster size of extremes Coles et al. (2001). In future studies, one could concentrate more on the persistence of extreme (and non-extreme) events in meridional energy transport based on the extremal index. The persistence of individual states together with the local attractor dimension can be used as a dynamical proxy of the general circulation. This approach has already successfully been applied to dynamical features of the atmosphere (Faranda et al., 2017; Messori et al., 2021)), but never, to the best of our knowledge, to thermodynamic features.

Finally, a possible outcome of this analysis, that is left for future work, is the study of meridional energy transport extremes, their zonal wavenumber decomposition and the underlying dynamical drivers, in numerical climate simulations. Assessing the statistics of these events against reanalysis-based data would provide an important background for the study of the climate response to external forcing and how changing statistics in extreme events related to baroclinic eddies can affect future weather predictability in the mid-latitudes (cfr. Scher and Messori (2019)). 
Data availability. ERA5 Reanalysis data are publicly accessible via Copernicus Data Storage. The computation of meridional energy transport and its wavenumber decomposition was performed at the ECMWF data server and stored at the Nird storage facility provided by the Norwegian e-infrastructure for research and education UNINETT Sigma2 under the project NS9063K.

\section{Appendix A: EOF patterns for k-means clusters selection}

Figures A1 and A2 show patterns of the first 4 EOFs for DJF and JJA, respectively. These have been used to obtain the fractional change in the PC mean described in Figure 4.

\section{Appendix B: Shifts in the extreme event PCs}

We investigate here changes in the PCs of the population of extremes, compared to the climatology. For the sake of simplicity, we restrict ourselves to the 4 leading EOFs, computed in DJF and JJA over the three regions of interest: EAT, PAC and NH. Fractional changes in the mean of the PC distribution relative to the climatological standard deviation (Figure B1) are evidenced, when significant according to Welch's T-test. We investigate shifts in the mean of the distribution in each dimension (PC), relative to the standard deviation of the climatological distribution.

In DJF, significant shifts are found for the first PC, corresponding to the positive phase of the Arctic Oscillation (AO) pattern for NH (Thompson and Wallace, 1998) and to the corresponding local patterns for EAT (NAO+) and PAC (Bering Ridge) (see Fig. A1 in Appendix A). Consistently, the distributions shift in opposite directions for equator- and pole-ward transport extremes respectively. The distribution of the third PCs (denoting a positive geopotential anomaly over the North Atlantic in EAT and over eastern Siberia in PAC) also change significantly in the population of equatorward extremes. In JJA, the most significant changes occur for higher order EOFs, with the only exception of the poleward extremes in EAT, connected to the first EOF (NAO-). These higher order EOFs are typically associated with localized patterns (e.g. EOF 3 and 4 for EAT and NH in Fig. A2), indicating that the extremes in this season are related to smaller scales of the circulation.

Author contributions. VLe and GM designed the analysis and wrote the manuscript. RG performed the wavenumber decomposition of meridional energy transports, VMG conceived the algorithm for extreme events detection and carried out the convergence analysis, FF performed the EOF and k-means clustering analysis and attribution of events. All authors contributed to the interpretation of the results.

Competing interests. The authors declare that they have no competing interests. 
https://doi.org/10.5194/wcd-2021-85

Preprint. Discussion started: 4 January 2022

(C) Author(s) 2022. CC BY 4.0 License.

(c) (1)

Weather and

Climate Dynamics

Discussions

Acknowledgements. G. Messori has received funding from the European Research Council (ERC) under the European Union's Horizon 2020 research and innovation programme (Grant agreement No. 948309, CENÆ project). V. Lucarini acknowledges the support received

430 from the EPSRC project EP/T018178/1 and from the EU Horizon 2020 project TiPES (grant no. 820970). The work is also associated with the Norwegian Science Foundation (NFR) project No. 280727. 
https://doi.org/10.5194/wcd-2021-85

Preprint. Discussion started: 4 January 2022

(C) Author(s) 2022. CC BY 4.0 License.
Weather and

Climate Dynamics

Discussions

\section{References}

Ambaum, M. H. P.: Thermal Physics of the Atmosphere, Wiley-Blackwell, https://doi.org/10.1002/9780470710364, 2010.

Baggett, C. and Lee, S.: Arctic Warming Induced by Tropically Forced Tapping of Available Potential Energy and the Role of the Planetary-

Scale Waves, Journal of the Atmospheric Sciences, p. 150217142336005, https://doi.org/10.1175/JAS-D-14-0334.1, 2015.

Balkema, A. A. and Haan, L. d.: Residual Life Time at Great Age, The Annals of Probability, 2, pp. 792-804, https://doi.org/10.1214/aop/1176996548, 1974.

Branstator, G.: Circumglobal teleconnections, the jet stream waveguide, and the North Atlantic Oscillation, Journal of Climate, 15, 1893$1910,2002$.

Cassou, C.: Intraseasonal interaction between the Madden-Julian Oscillation and the North Atlantic Oscillation, Nature, 455, 523-527, https://doi.org/10.1038/nature07286, 2008.

Coles, S., Bawa, J., Trenner, L., and Dorazio, P.: An introduction to statistical modeling of extreme values, vol. 208, Springer, 2001.

Corti, S., Molteni, F., and Palmer, T. N.: Signature of recent climate change in frequencies of natural atmospheric circulation regimes, Nature, 398, 799-802, https://doi.org/10.1038/19745, 1999.

Coumou, D., Petoukhov, V., Rahmstorf, S., Petri, S., and Schellnhuber, H. J.: Quasi-resonant circulation regimes and hemispheric synchronization of extreme weather in boreal summer, Proceedings of the National Academy of Sciences, 111, 12 331-12 336, 2014.

Davini, P. and D'Andrea, F.: Northern Hemisphere Atmospheric Blocking Representation in Global Climate Models: Twenty Years of Improvements?, Journal of Climate, 29, 8823-8840, https://doi.org/10.1175/JCLI-D-16-0242.1, 2016.

Dawson, A., Palmer, T. N., and Corti, S.: Simulating regime structures in weather and climate prediction models, Geophysical Research Letters, 39, https://doi.org/https://doi.org/10.1029/2012GL053284, 2012.

Dole, R., Hoerling, M., Perlwitz, J., Eischeid, J., Pegion, P., Zhang, T., Quan, X.-W., Xu, T., and Murray, D.: Was there a basis for anticipating the 2010 Russian heat wave?, Geophysical Research Letters, 38, https://doi.org/https://doi.org/10.1029/2010GL046582, 2011.

Fabiano, F., Christensen, H., Strommen, K., Athanasiadis, P., Baker, A., Schiemann, R., and Corti, S.: Euro-Atlantic weather Regimes in the PRIMAVERA coupled climate simulations: impact of resolution and mean state biases on model performance, Climate Dynamics, 54 , 5031-5048, 2020.

Fabiano, F., Meccia, V. L., Davini, P., Ghinassi, P., and Corti, S.: A regime view of future atmospheric circulation changes in northern mid-latitudes, Weather and Climate Dynamics, 2, 163-180, https://doi.org/10.5194/WCD-2-163-2021, 2021.

Faranda, D., Messori, G., and Yiou, P.: Dynamical proxies of North Atlantic predictability and extremes, Scientific reports, 7, 1-10, 2017.

Ferro, C. A. T. and Segers, J.: Inference for Clusters of Extreme Values, Journal of the Royal Statistical Society. Series B (Statistical Methodology), 65, 545-556, http://www.jstor.org/stable/3647520, 2003.

Forthofer, R. N. and Lehnen, R. G.: Rank Correlation Methods, Public Program Analysis, pp. 146-163, https://doi.org/10.1007/978-1-46846683-6_9, 1981.

Galfi, V. M. and Lucarini, V.: Fingerprinting Heatwaves and Cold Spells and Assessing Their Response to Climate Change Using Large Deviation Theory, Phys. Rev. Lett., 127, 058 701, https://doi.org/10.1103/PhysRevLett.127.058701, 2021.

465 Gálfi, V. M., Bódai, T., and Lucarini, V.: Convergence of extreme value statistics in a two-layer quasi-geostrophic atmospheric model, Complexity, 2017, 2017.

Gálfi, V. M., Lucarini, V., and Wouters, J.: A large deviation theory-based analysis of heat waves and cold spells in a simplified model of the general circulation of the atmosphere, Journal of Statistical Mechanics: Theory and Experiment, 2019, 033 404, 2019. 
https://doi.org/10.5194/wcd-2021-85

Preprint. Discussion started: 4 January 2022

(C) Author(s) 2022. CC BY 4.0 License.
Weather and

Climate Dynamics

Discussions

Gálfi, V. M., Lucarini, V., Ragone, F., and Wouters, J.: Applications of large deviation theory in geophysical fluid dynamics and climate science, La Rivista del Nuovo Cimento, 44, 291-363, https://doi.org/10.1007/s40766-021-00020-z, 2021.

Gnedenko, B.: Sur la distribution limite du terme maximum d'une serie aleatoire, Annals of mathematics, pp. 423-453, 1943.

Graversen, R. G. and Burtu, M.: Arctic amplification enhanced by latent energy transport of atmospheric planetary waves, Quarterly Journal of the Royal Meteorological Society, 142, 2046-2054, 2016.

Guemas, V., Salas-Mélia, D., Kageyama, M., Giordani, H., Voldoire, A., and Sanchez-Gomez, E.: Summer interactions between weather regimes and surface ocean in the North-Atlantic region, Climate Dynamics, 34, 527-546, https://doi.org/10.1007/s00382-008-0491-6, 2010 .

Hannachi, A., Straus, D. M., Franzke, C. L. E., Corti, S., and Woollings, T.: Low-frequency nonlinearity and regime behavior in the Northern Hemisphere extratropical atmosphere, Reviews of Geophysics, 55, 199-234, https://doi.org/10.1002/2015RG000509, 2017.

Heiskanen, T., Graversen, R. G., Rydsaa, J. H., and Isachsen, P. E.: Comparing wavelet and Fourier perspectives on the decomposition of meridional energy transport into synoptic and planetary components, Quarterly Journal of the Royal Meteorological Society, 146, 2717-2730, https://doi.org/10.1002/QJ.3813, 2020.

Hersbach, H., Bell, B., Berrisford, P., Hirahara, S., Horányi, A., Muñoz-Sabater, J., Nicolas, J., Peubey, C., Radu, R., Schepers, D., et al.: The ERA5 global reanalysis, Quarterly Journal of the Royal Meteorological Society, 146, 1999-2049, 2020.

Hochman, A., Messori, G., Quinting, J. F., Pinto, J. G., and Grams, C. M.: Do Atlantic-European weather regimes physically exist?, Geophysical Research Letters, p. e2021GL095574, 2021.

Holton, J. R. and Hakim, G. J.: An Introduction to Dynamic Meteorology, Academic Press, 2012.

Jung, T., Palmer, T. N., and Shutts, G. J.: Influence of a stochastic parameterization on the frequency of occurrence of North Pacific weather regimes in the ECMWF model, Geophysical Research Letters, 32, https://doi.org/https://doi.org/10.1029/2005GL024248, 2005.

Kaspi, Y. and Schneider, T.: The role of stationary eddies in shaping midlatitude storm tracks, Journal of the atmospheric sciences, 70 , 2596-2613, 2013.

Kimoto, M. and Ghil, M.: Multiple Flow Regimes in the Northern Hemisphere Winter. Part I: Methodology and Hemispheric Regimes, Journal of Atmospheric Sciences, 50, 2625 - 2644, https://doi.org/10.1175/1520-0469(1993)050<2625:MFRITN>2.0.CO;2, 1993.

Kornhuber, K., Petoukhov, V., Petri, S., Rahmstorf, S., and Coumou, D.: Evidence for wave resonance as a key mechanism for generating high-amplitude quasi-stationary waves in boreal summer, Climate Dynamics, 49, 1961-1979, 2017.

Kornhuber, K., Coumou, D., Vogel, E., Lesk, C., Donges, J. F., Lehmann, J., and Horton, R. M.: Amplified Rossby waves enhance risk of concurrent heatwaves in major breadbasket regions, Nature Climate Change, 10, 48-53, 2020.

Leadbetter, M., Weissman, I., De Haan, L., and Rootzén, H.: On clustering of high values in statistically stationary series, Proc. 4th Int. Meet. Statistical Climatology, 16, 217-222, 1989.

Leadbetter, M. R.: On extreme values in stationary sequences, Zeitschrift für Wahrscheinlichkeitstheorie und verwandte Gebiete, 28, 289303, 1974.

Lembo, V., Messori, G., Graversen, R. G., and Lucarini, V.: Spectral Decomposition and Extremes of Atmospheric Meridional Energy Transport in the Northern Hemisphere Midlatitudes, Geophysical Research Letters, 46, 2019GL082 105, https://doi.org/10.1029/2019GL082105, 2019.

Liang, M., Czaja, A., Graversen, R., and Tailleux, R.: Poleward energy transport: is the standard definition physically relevant at all time scales?, Climate Dynamics 2017 50:5, 50, 1785-1797, https://doi.org/10.1007/S00382-017-3722-X, 2017. 
https://doi.org/10.5194/wcd-2021-85

Preprint. Discussion started: 4 January 2022

(c) Author(s) 2022. CC BY 4.0 License.

(c) (i)

Weather and

Climate Dynamics

Discussions

Lorenz, E. N.: Available Potential Energy and the Maintenance of the General Circulation, Tellus, 7, 157-167, https://doi.org/10.1111/j.21533490.1955.tb01148.x, 1955.

Lorenz, E. N.: The nature and theory of the general circulation of the atmosphere, vol. 218, World Meteorological Organization Geneva, 1967.

510 Lucarini, V. and Gritsun, A.: A new mathematical framework for atmospheric blocking events, Climate Dynamics, 54, 575-598, https://doi.org/10.1007/S00382-019-05018-2/FIGURES/14, 2020.

Lucarini, V. and Ragone, F.: Energetics of Climate Models: Net Energy Balance and Meridional Enthalpy Transport, Reviews of Geophysics, 49, https://doi.org/10.1029/2009RG000323, 2011.

Mann, H.: Nonparametric tests against trend, Econometrica, 13, 245-259, 1945.

515 Messori, G. and Czaja, A.: On the sporadic nature of meridional heat transport by transient eddies, Quarterly Journal of the Royal Meteorological Society, 139, 999-1008, 2013.

Messori, G. and Czaja, A.: Some considerations on the spectral features of meridional heat transport by transient eddies, Quarterly Journal of the Royal Meteorological Society, 140, 1377-1386, 2014.

Messori, G. and Czaja, A.: On local and zonal pulses of atmospheric heat transport in reanalysis data, Quarterly Journal of the Royal Meteorological Society, 141, 2376-2389, 2015.

Messori, G., Geen, R., and Czaja, A.: On the spatial and temporal variability of atmospheric heat transport in a hierarchy of models, Journal of the Atmospheric Sciences, 74, 2163-2189, 2017.

Messori, G., Woods, C., and Caballero, R.: On the drivers of wintertime temperature extremes in the high Arctic, Journal of Climate, 31, 1597-1618, 2018.

Messori, G., Harnik, N., Madonna, E., Lachmy, O., and Faranda, D.: A dynamical systems characterization of atmospheric jet regimes, Earth System Dynamics, 12, 233-251, 2021.

Michelangeli, P.-A., Vautard, R., and Legras, B.: Weather Regimes: Recurrence and Quasi Stationarity, Journal of Atmospheric Sciences, 52, 1237-1256, https://doi.org/10.1175/1520-0469(1995)052<1237:WRRAQS>2.0.CO;2, 1995.

Nie, J., Wang, P., Yang, W., and Tan, B.: Northern hemisphere storm tracks in strong AO anomaly winters, Atmospheric Science Letters, 9 , 153-159, https://doi.org/10.1002/ASL.186, 2008.

Novak, L., Ambaum, M. H. P., and Tailleux, R.: The Life Cycle of the North Atlantic Storm Track, Journal of Atmospheric Sciences, 72, 821-833, https://doi.org/10.1175/JAS-D-14-0082.1, 2015.

Papritz, L.: Arctic lower-tropospheric warm and cold extremes: Horizontal and vertical transport, diabatic processes, and linkage to synoptic circulation features, Journal of Climate, 33, 993-1016, 2020.

Pasquier, J., Pfahl, S., and Grams, C. M.: Modulation of atmospheric river occurrence and associated precipitation extremes in the North Atlantic region by European weather regimes, Geophysical Research Letters, 46, 1014-1023, 2019.

Peixoto, J. P. and Oort, A. H.: Physics of climate, New York, NY (United States); American Institute of Physics, 1992.

Petoukhov, V., Rahmstorf, S., Petri, S., and Schellnhuber, H. J.: Quasiresonant amplification of planetary waves and recent Northern Hemisphere weather extremes, Proceedings of the National Academy of Sciences, 110, 5336-5341, 2013.

540 Pickands, J. I.: Statistical Inference Using Extreme Order Statistics, The Annals of Statistics, 3, 119-131, https://doi.org/10.1214/aos/1176343003, 1975.

Ragone, F. and Bouchet, F.: Computation of Extreme Values of Time Averaged Observables in Climate Models with Large Deviation Techniques, Journal of Statistical Physics, 179, 1637-1665, https://doi.org/10.1007/S10955-019-02429-7/FIGURES/10, 2020. 
https://doi.org/10.5194/wcd-2021-85

Preprint. Discussion started: 4 January 2022

(c) Author(s) 2022. CC BY 4.0 License.

(c) (i)

Weather and

Climate Dynamics

Discussions

Ragone, F. and Bouchet, F.: Rare Event Algorithm Study of Extreme Warm Summers and Heatwaves Over Europe, Geophysical Research Letters, 48, e2020GL091 197, https://doi.org/10.1029/2020GL091197, 2021.

Ruggieri, P., Alvarez-Castro, M. C., Athanasiadis, P., Bellucci, A., Materia, S., and Gualdi, S.: North Atlantic circulation regimes and heat transport by synoptic eddies, Journal of Climate, 33, 4769-4785, https://doi.org/10.1175/JCLI-D-19-0498.1, 2020.

Scher, S. and Messori, G.: How Global Warming Changes the Difficulty of Synoptic Weather Forecasting, Geophysical Research Letters, 46, 2931-2939, https://doi.org/https://doi.org/10.1029/2018GL081856, 2019.

550 Schiemann, R., Athanasiadis, P., Barriopedro, D., Doblas-Reyes, F., Lohmann, K., Roberts, M. J., Sein, D. V., Roberts, C. D., Terray, L., and Vidale, P. L.: Northern Hemisphere blocking simulation in current climate models: evaluating progress from the Climate Model Intercomparison Project Phase 5 to 6 and sensitivity to resolution, Weather and Climate Dynamics, 1, 277-292, https://doi.org/10.5194/wcd-1-2772020,2020

Starr, V. and White, R.: Balance Requirements of the General Circulation, Tech. rep., Air Force Research Center: Cambridge, MA, https: //apps.dtic.mil/sti/citations/AD0059398, 1954

Straus, D. M., Molteni, F., and Corti, S.: Atmospheric Regimes: The Link between Weather and the Large-Scale Circulation, p. 105-135, Cambridge University Press, https://doi.org/10.1017/9781316339251.005, 2017.

Strommen, K., Mavilia, I., Corti, S., Matsueda, M., Davini, P., von Hardenberg, J., Vidale, P.-L., and Mizuta, R.: The Sensitivity of Euro-Atlantic Regimes to Model Horizontal Resolution, Geophysical Research Letters, 46, 7810-7818, https://doi.org/https://doi.org/10.1029/2019GL082843, 2019.

Swanson, K. L. and Pierrehumbert, R. T.: Lower-tropospheric heat transport in the Pacific storm track, Journal of the atmospheric sciences, 54, 1533-1543, 1997.

Thompson, D. W. and Wallace, J. M.: The Arctic Oscillation signature in the wintertime geopotential height and temperature fields, Geophysical research letters, 25, 1297-1300, 1998.

Weisheimer, A., Corti, S., Palmer, T., and Vitart, F.: Addressing model error through atmospheric stochastic physical parametrizations: impact on the coupled ECMWF seasonal forecasting system, Philosophical Transactions of the Royal Society A: Mathematical, Physical and Engineering Sciences, 372, https://doi.org/10.1098/RSTA.2013.0290, 2014.

Woollings, T., Barriopedro, D., Methven, J., Son, S. W., Martius, O., Harvey, B., Sillmann, J., Lupo, A. R., and Seneviratne, S.: Blocking and its Response to Climate Change, Current Climate Change Reports 2018 4:3, 4, 287-300, https://doi.org/10.1007/S40641-018-0108-Z, 2018.

Yiou, P., Malamud, B. D., and Rust, H. W.: Preface "Extreme Events: Nonlinear Dynamics and Time Series Analysis", Nonlinear Processes in Geophysics, 18, 895-897, https://doi.org/10.5194/npg-18-895-2011, 2011. 

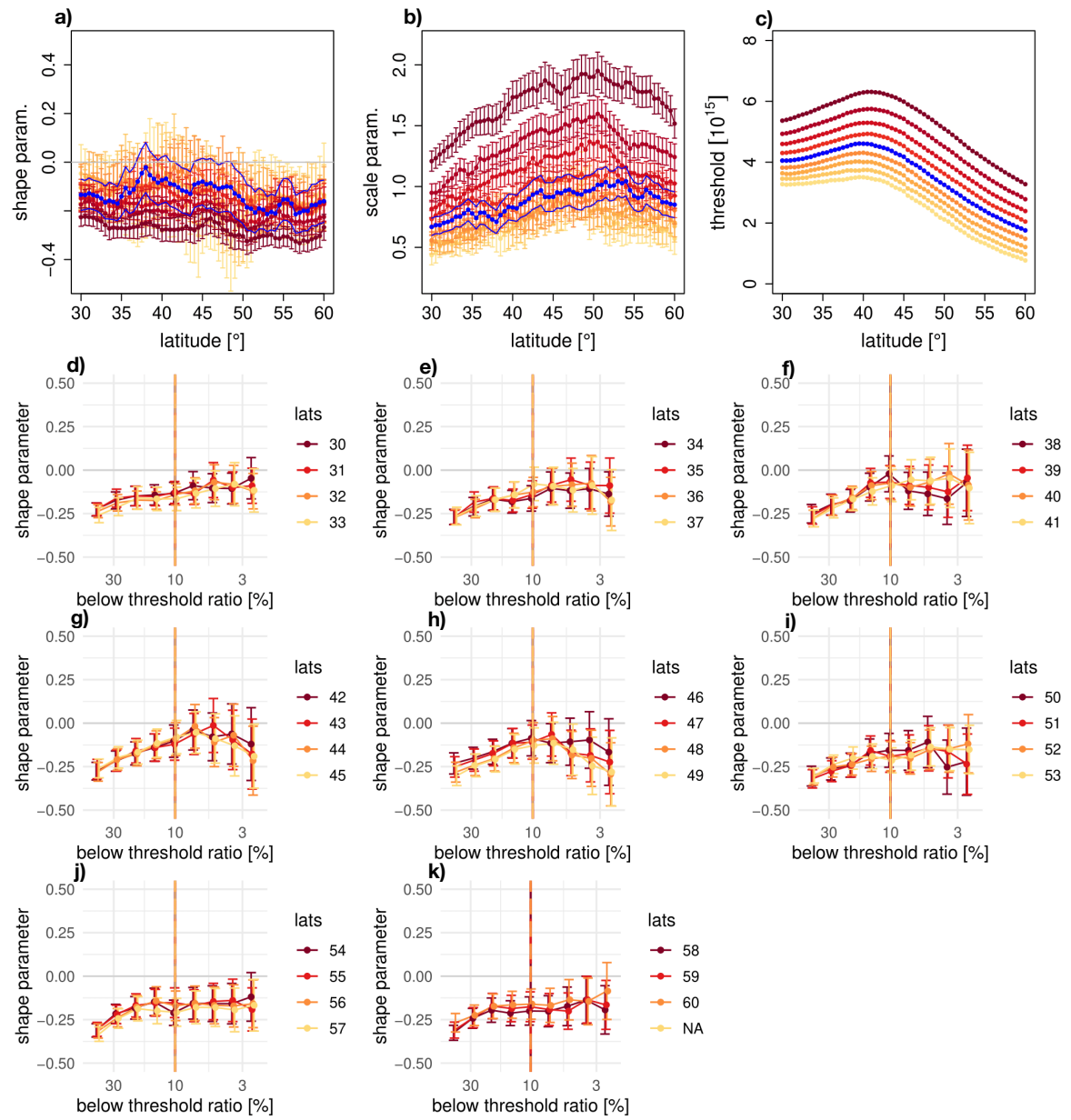

lats

$\rightarrow 58$

$\rightarrow 59$

$\rightarrow 60$

$\rightarrow$ NA

Figure 1. (Top) Meridional section for equatorward DJF meridional energy transport extremes of: a. Shape parameter $\xi$; b. Scale parameter $\sigma$; c. Threshold value $\left[10^{15} \mathrm{~W}\right]$. The colours in a and b denote parameter estimates corresponding to the threshold values shown in $\mathrm{c}$. The blue lines denote the final shape and scale parameter estimates based on the blue threshold values. (Bottom) Shape parameter as function of threshold (in terms of fraction of data points below the threshold) at different latitudes. The uncertainty range is defined based on $95 \%$ maximum likelihood confidence intervals. The orange vertical line denotes the selected fraction of data for the specific tail, which is chosen to be the same at all latitudes. 
https://doi.org/10.5194/wcd-2021-85

Preprint. Discussion started: 4 January 2022

(c) Author(s) 2022. CC BY 4.0 License.

(a) DJF, EAT
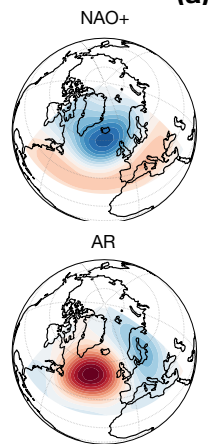

(c) DJF, PAC
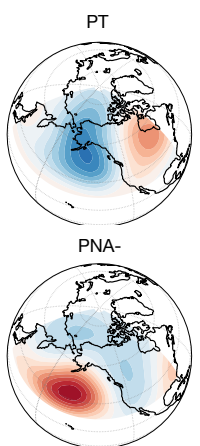

(e) DJF, NH
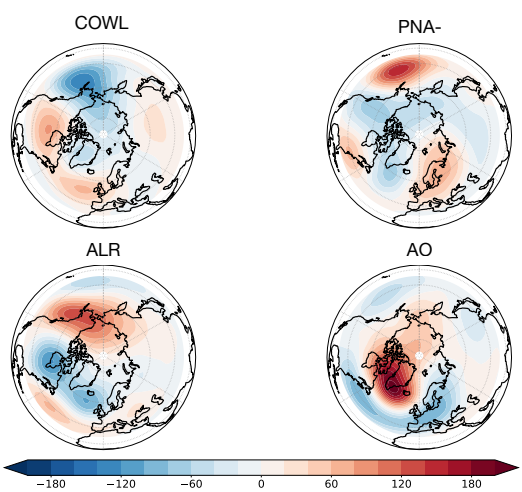

(b) JJA, EAT
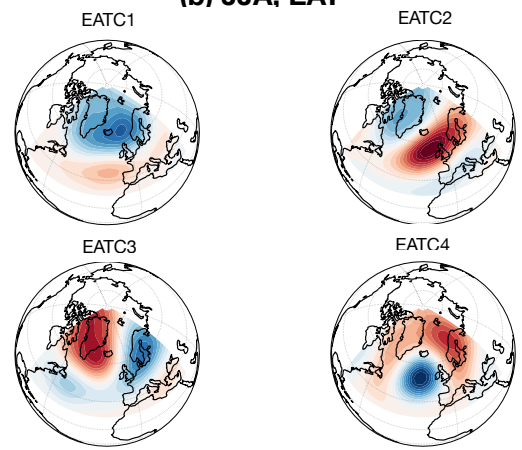

(d) JJA, PAC
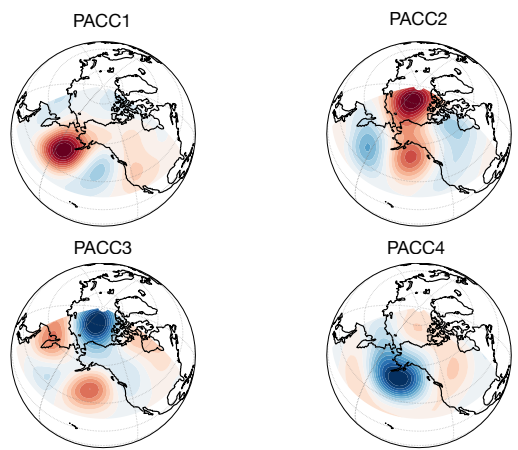

(f) JJA, NH
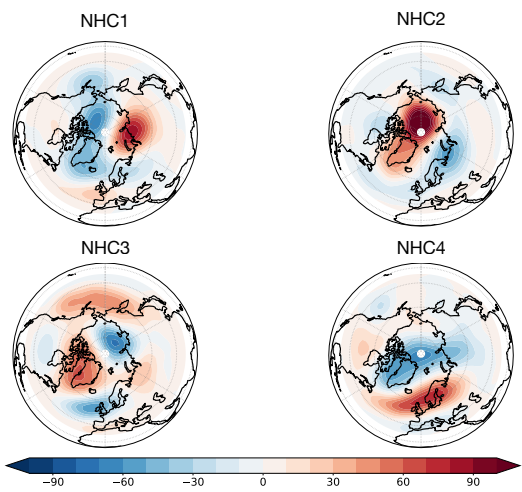

Figure 2. Clusters of zg500 geopotential height anomalies (in dam) by frequency in: (a) DJF, computed over the EAT region; (c) DJF, computed over the PAC region; (e) DJF, computed over the NH region; (b) JJA, computed over the EAT region; (d) JJA, computed over the PAC region; (f) JJA, computed over the NH region. 
(a) djf
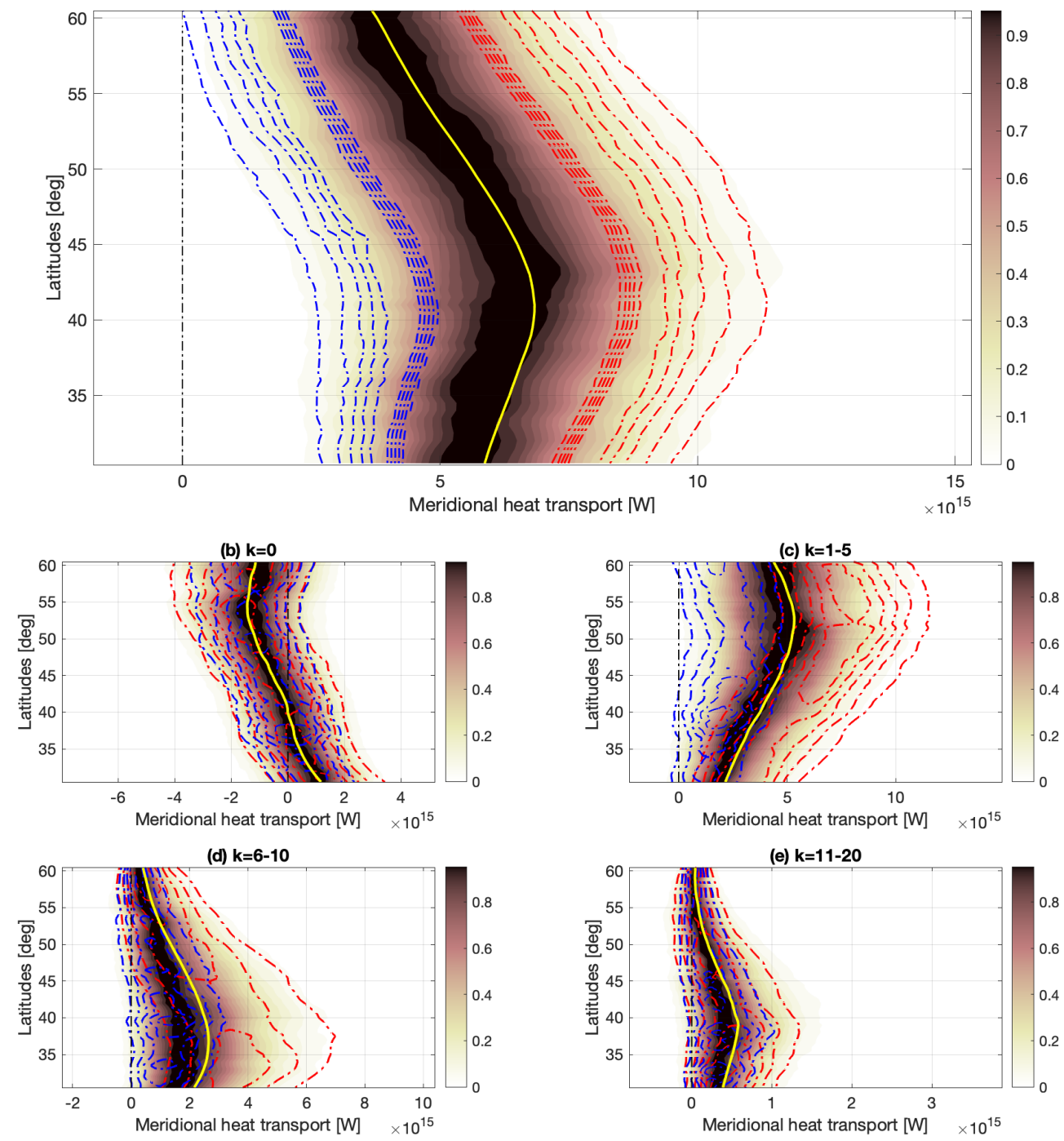

Figure 3. PDFs of total (filled contours) and extremes poleward (red contours) and equatorward (blue contours) DJF meridional energy transports over the 1979-2012 period in ERA5. (a) Sum of all wavenumber contributions; (b) k=0 (zonal mean); (c) k=1-5 (planetary scales); (d) k=6-10 (synoptic scales); (e) k=11-20 (higher scales). Total PDFs have been normalised at each latitude; PDFs of extremes are weighted at each latitude by the number of extreme events. Yellow lines denote mean values. 
https://doi.org/10.5194/wcd-2021-85

Preprint. Discussion started: 4 January 2022

(c) Author(s) 2022. CC BY 4.0 License.

(a) jja
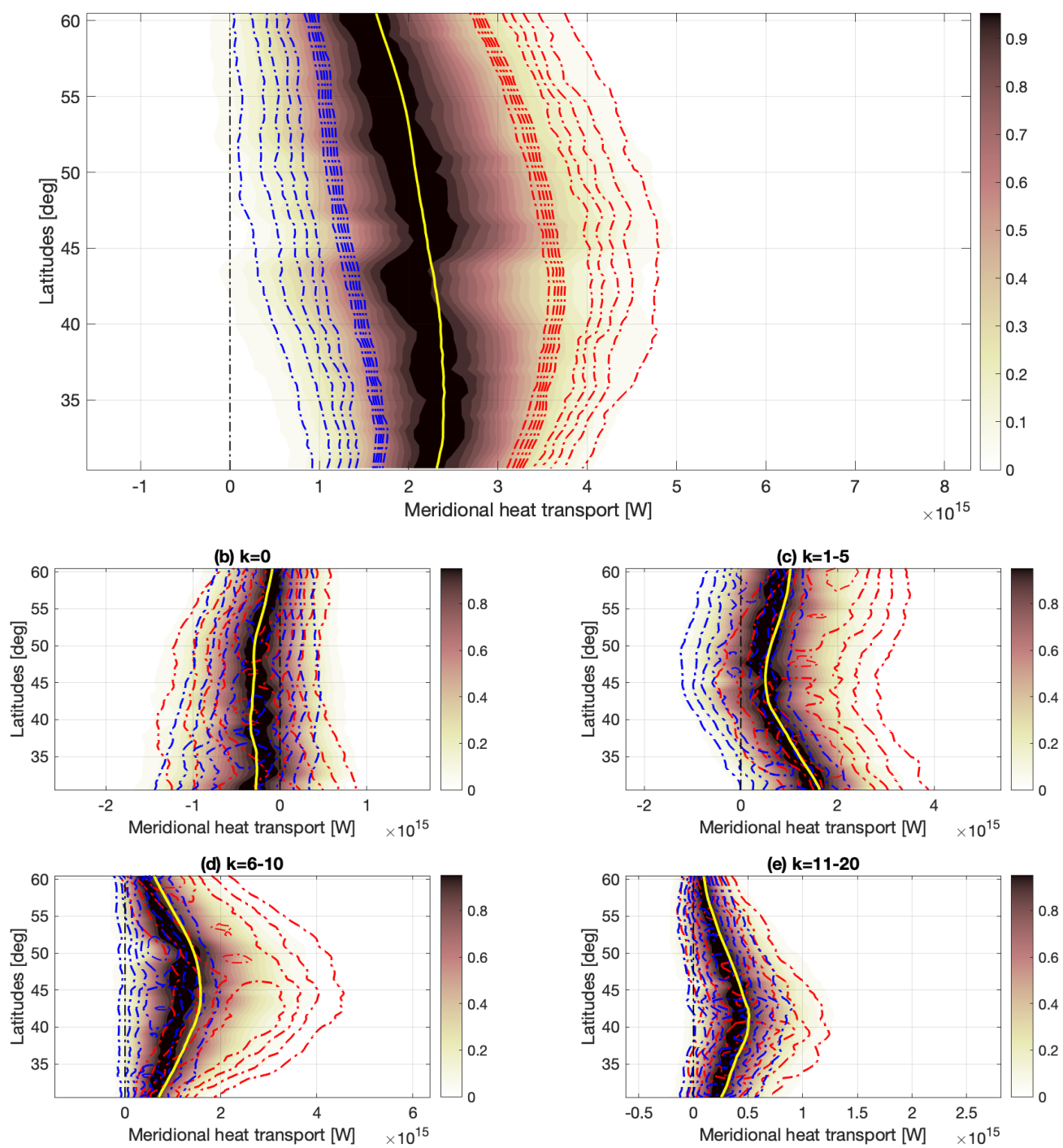

Figure 4. Same as in Figure 4, for JJA. 
(a)

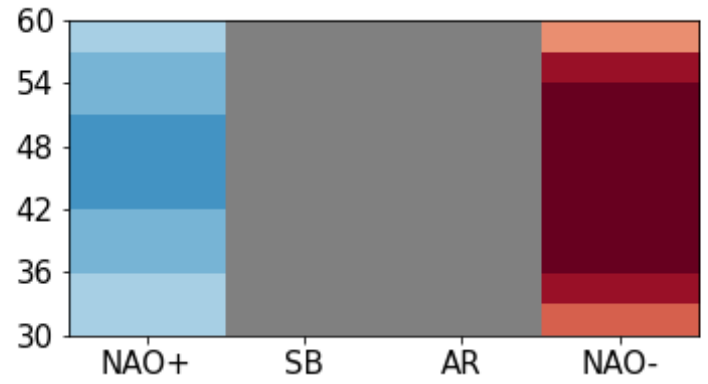

(c)

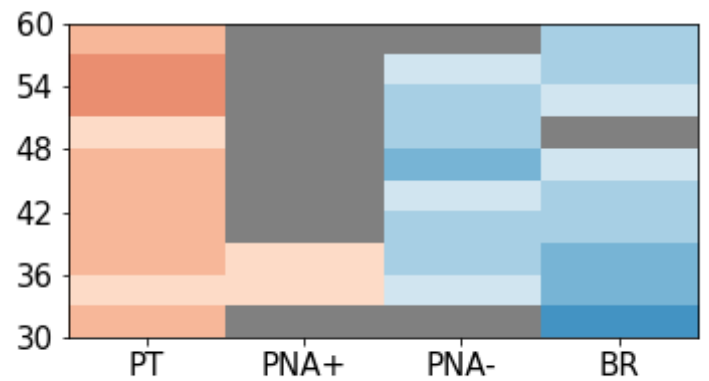

(e)

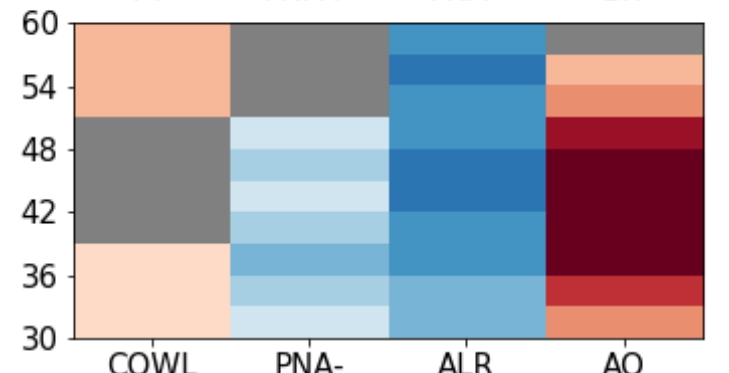

(b)

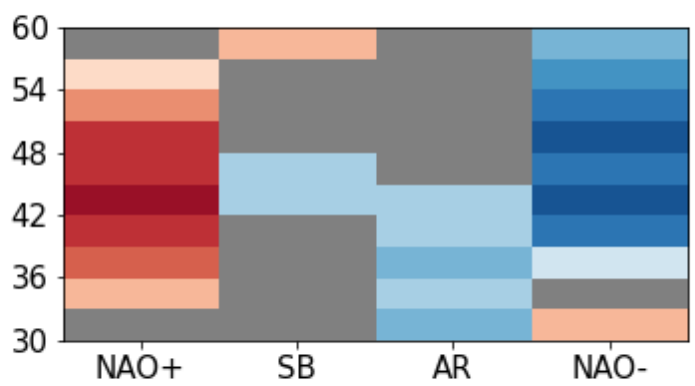

(d)

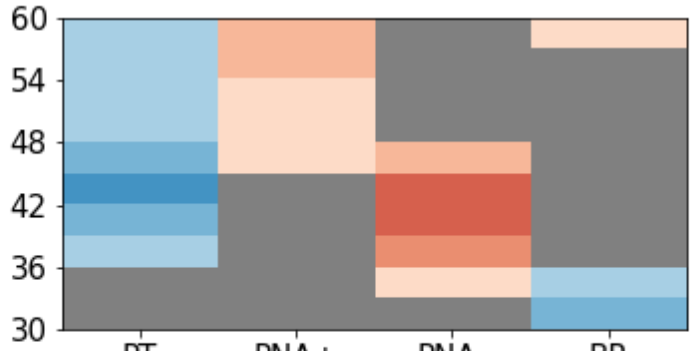

(f)

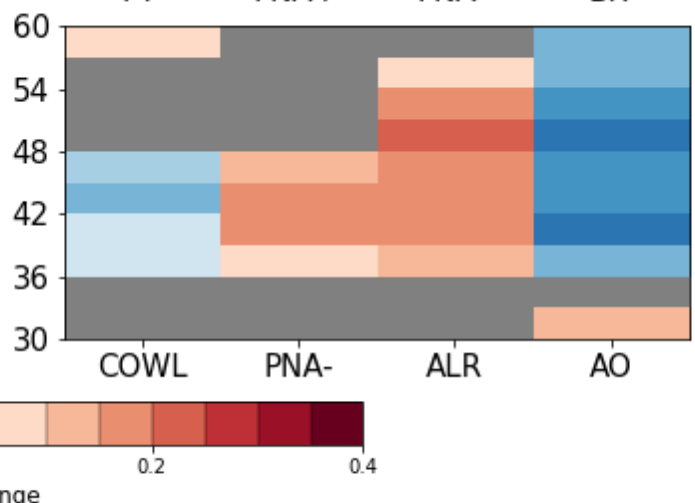

Figure 5. Relative variations in absolute frequency of clusters $\Delta f_{x}^{\prime}$ in the population of DJF extremes as a function of the latitude at which extremes are found: (a) EAT region, poleward extremes; (b) EAT region, equatorward extremes; (c) PAC region, poleward extremes; (d) PAC region, equartorward extremes; (e) NH region, poleward extremes; (f) NH region, equatorward extremes. Grey boxes denote non-significant frequency variations, according to the bootstrapping methodology described in the Methods section. 
(a)

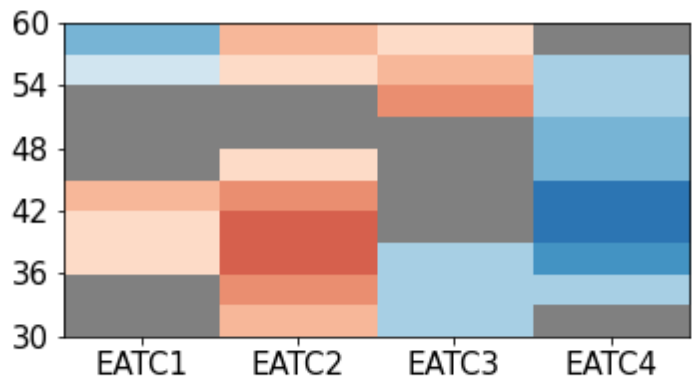

(c)

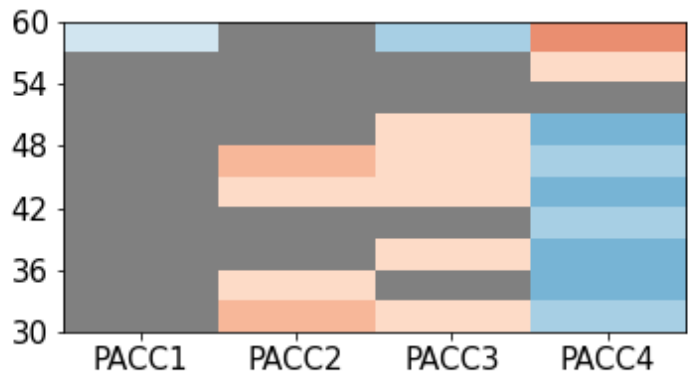

(e)
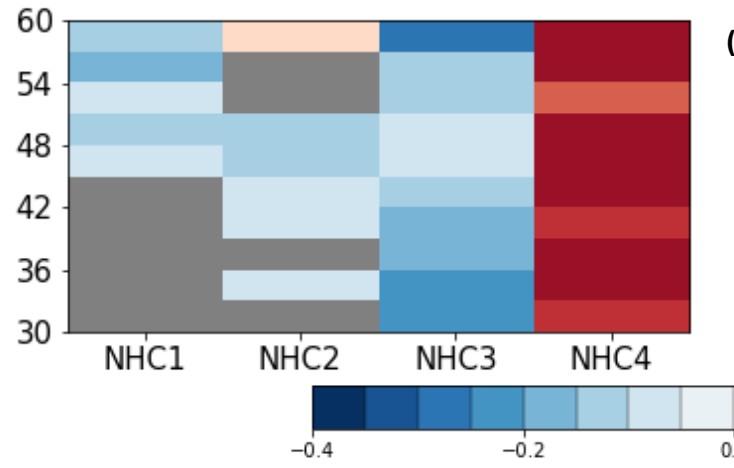

Abs. freq. change

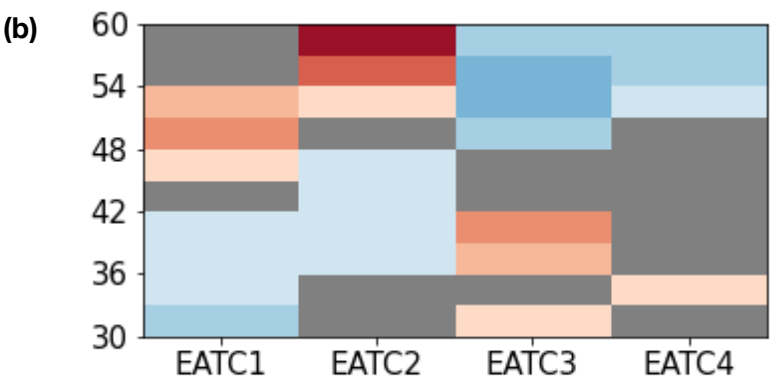

(d)

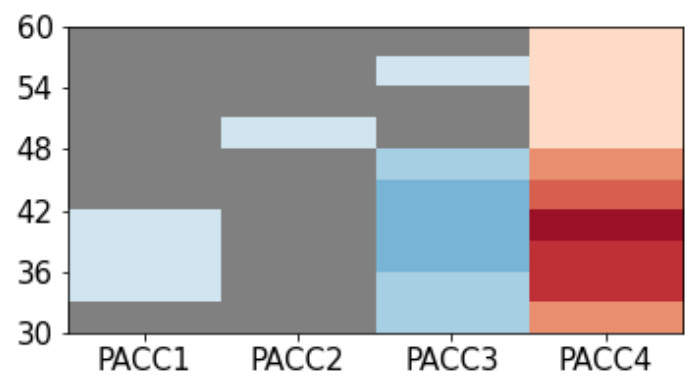

(f)

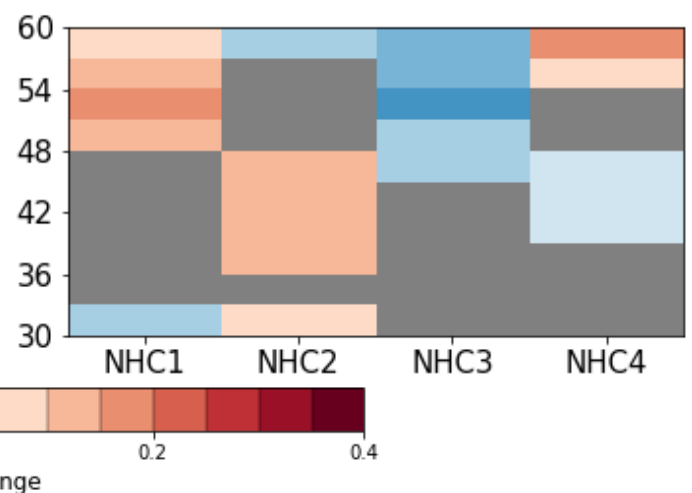

Figure 6. Same as in Figure 5, for JJA. 
https://doi.org/10.5194/wcd-2021-85

Preprint. Discussion started: 4 January 2022

(c) Author(s) 2022. CC BY 4.0 License.

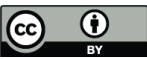

(a)

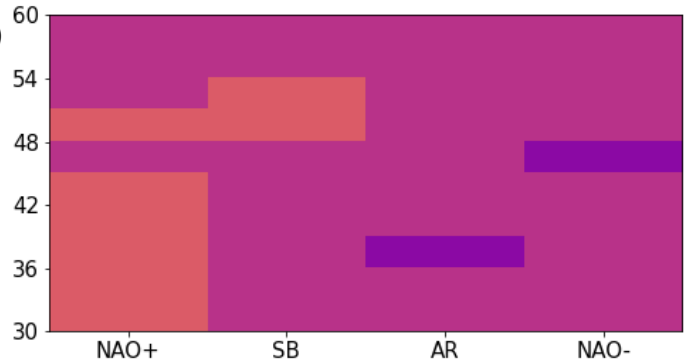

(c)

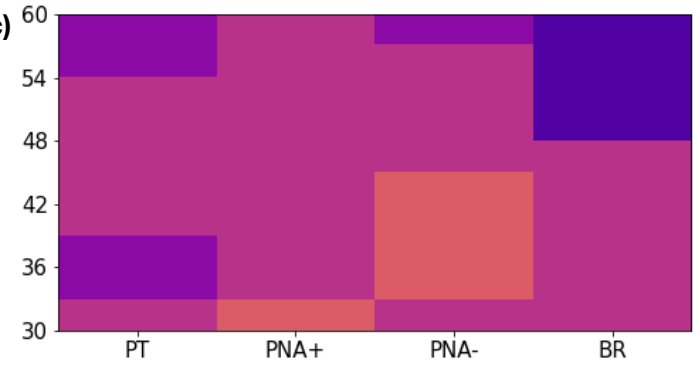

(e)

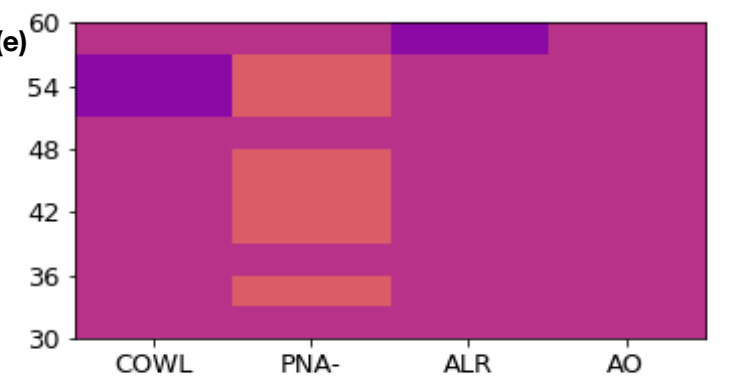

(b)

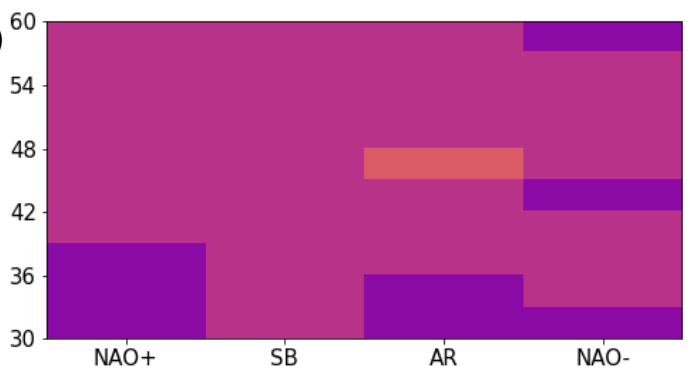

(d) 60

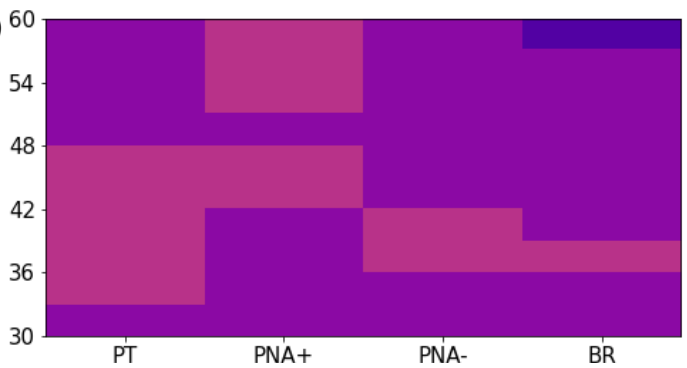

(f)

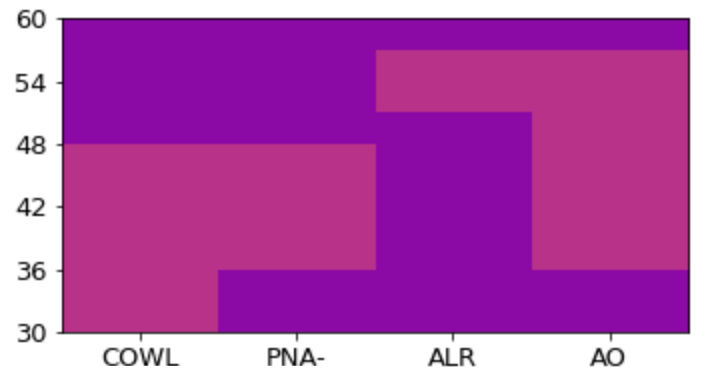

Figure 7. Time-averaged zonal wavenumber associated with meridional energy transport extremes (see text) for DJF clusters obtained in different regions, as a function of the latitude at which the extreme is found. (a) EAT region, poleward extremes; (b) EAT region, equatorward extremes; (c) PAC region, poleward extremes; (d) PAC region, equartorward extremes; (e) NH region, poleward extremes; (f) NH region, equatorward extremes. 
https://doi.org/10.5194/wcd-2021-85

Preprint. Discussion started: 4 January 2022

(c) Author(s) 2022. CC BY 4.0 License.

\section{(c) (i)}

(a)

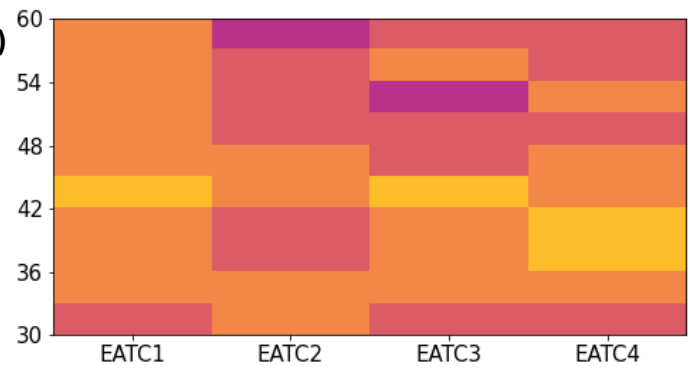

(c) 60

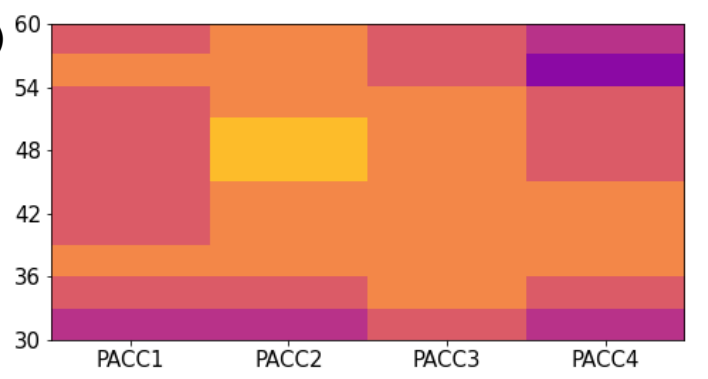

(e)
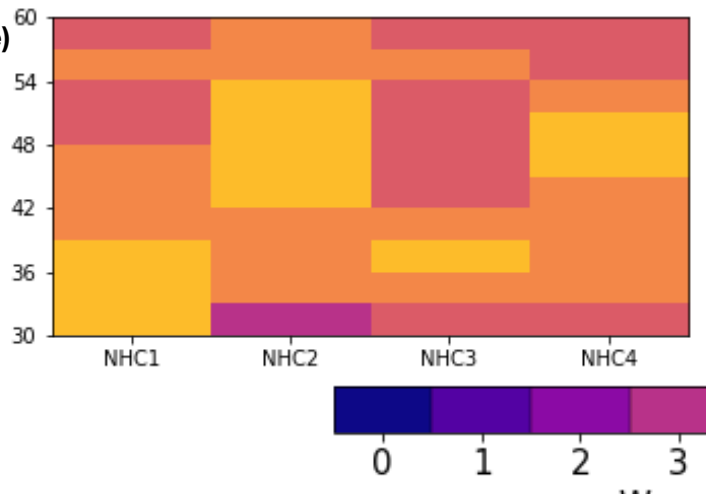

(b)

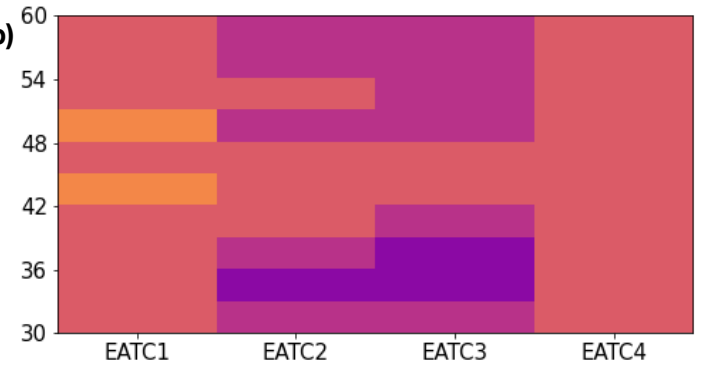

(d)

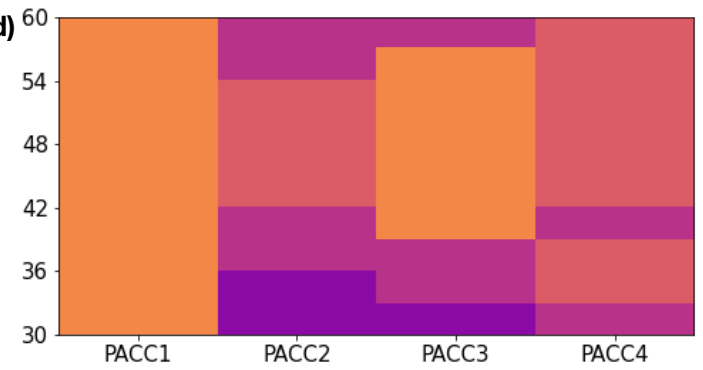

(f)

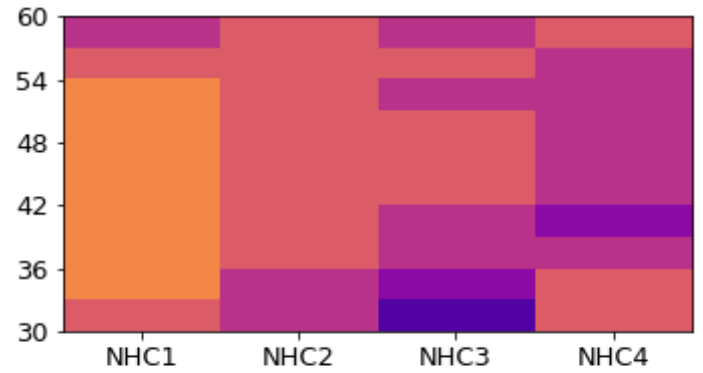

\section{Wavenumber}

Figure 8. Same as in Figure 7, for JJA. 


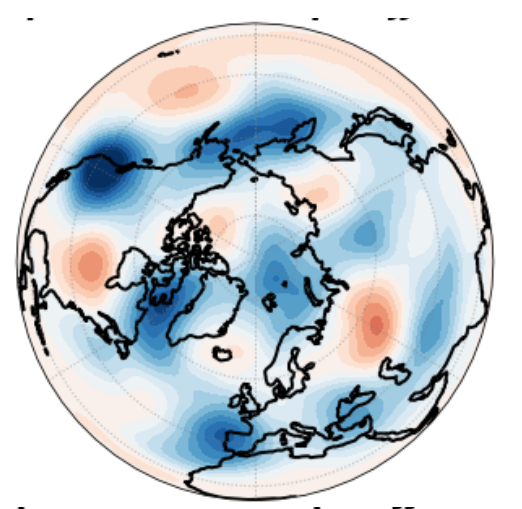

30-33
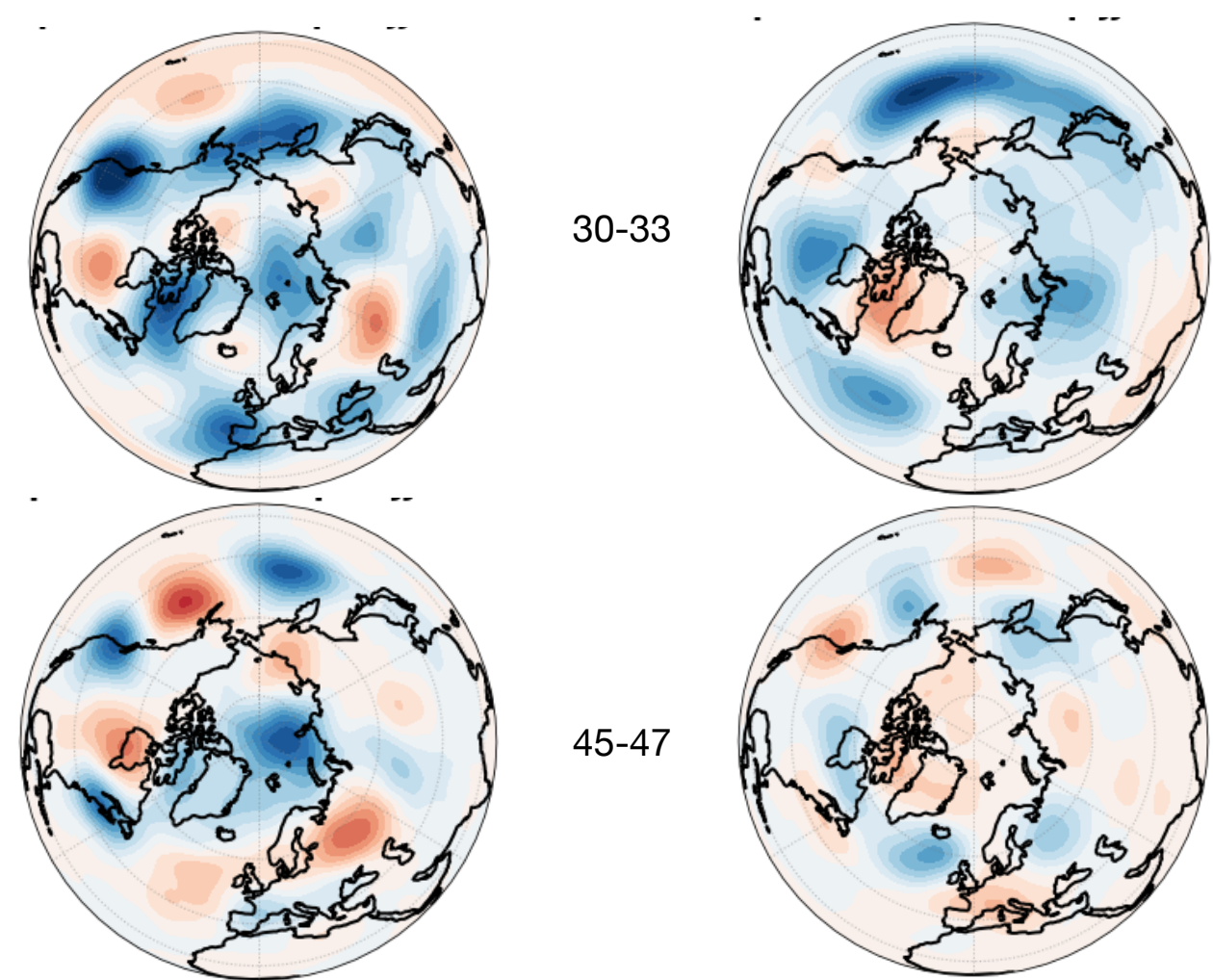

45-47
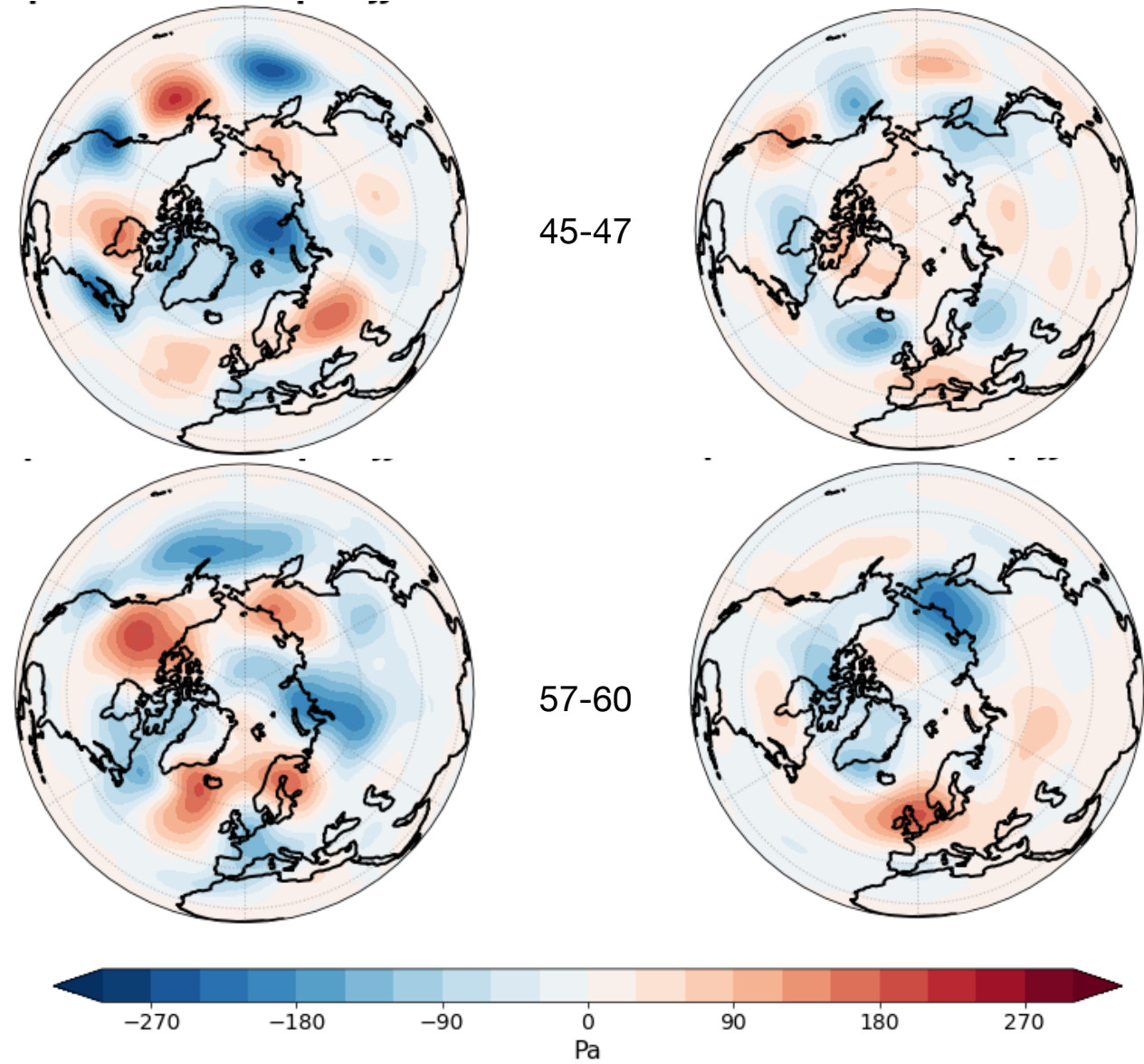

Figure 9. Composite mean of z500 anomalies (in $d a m$ ) for JJA (a) poleward and (b) equatorward meridional energy transport extremes at three chosen latitudinal bands. 


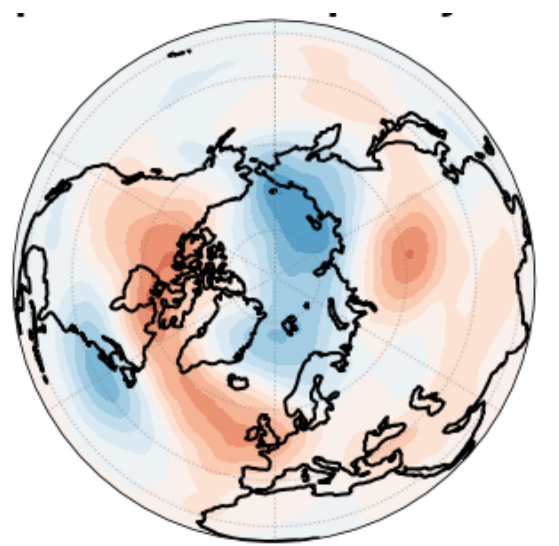

30-33

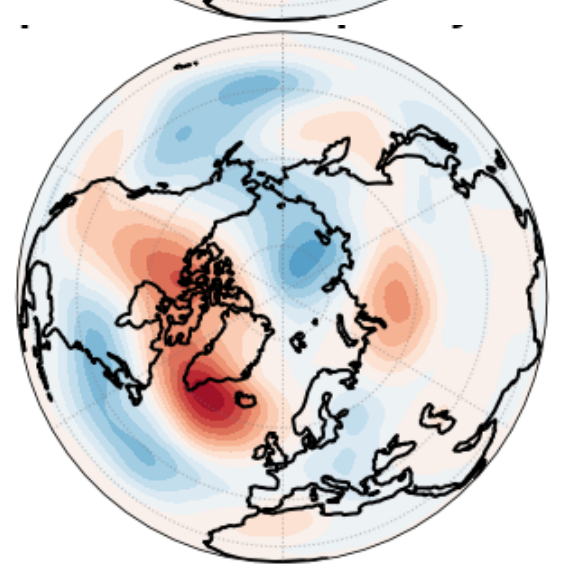

$45-47$
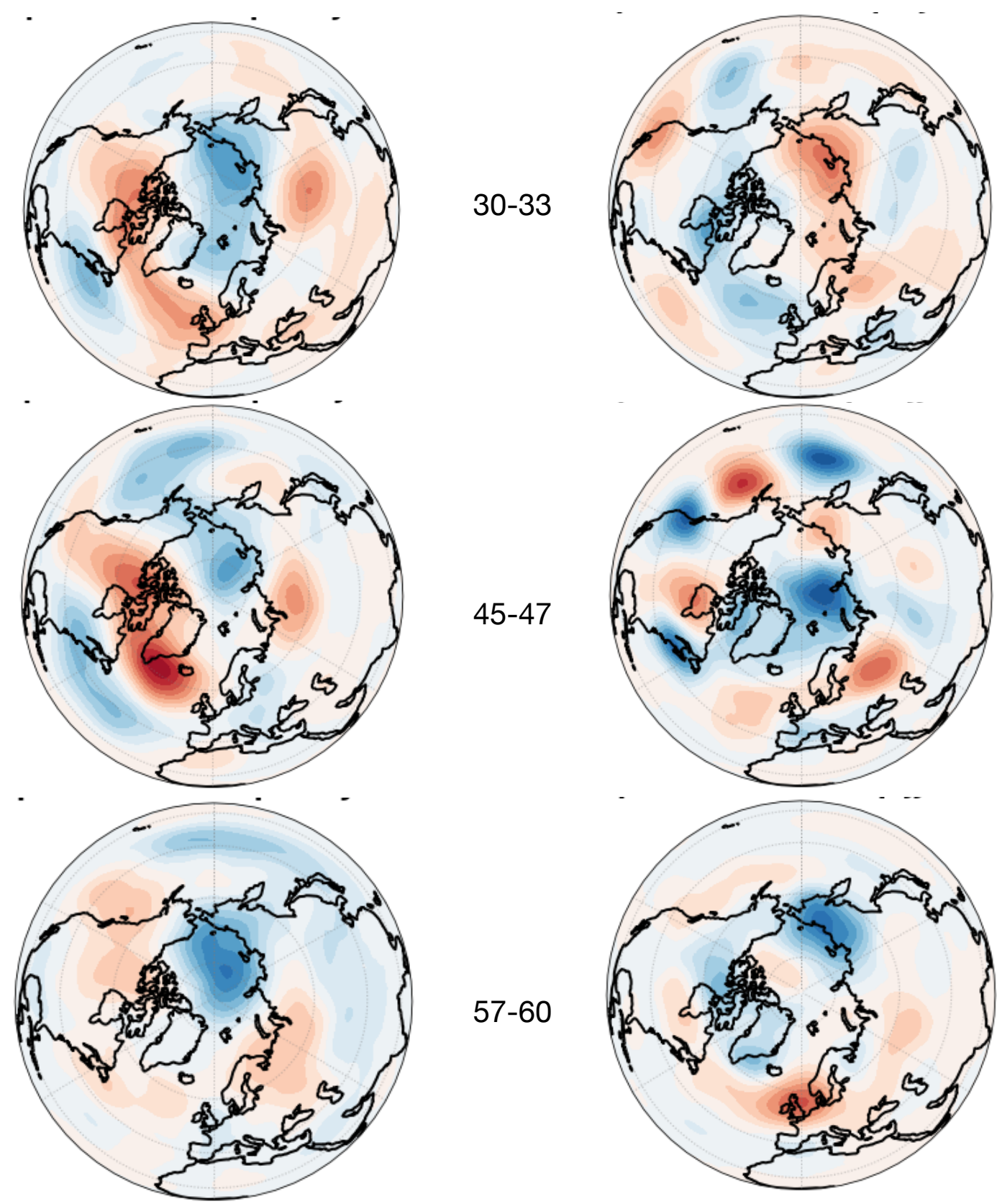

$57-60$
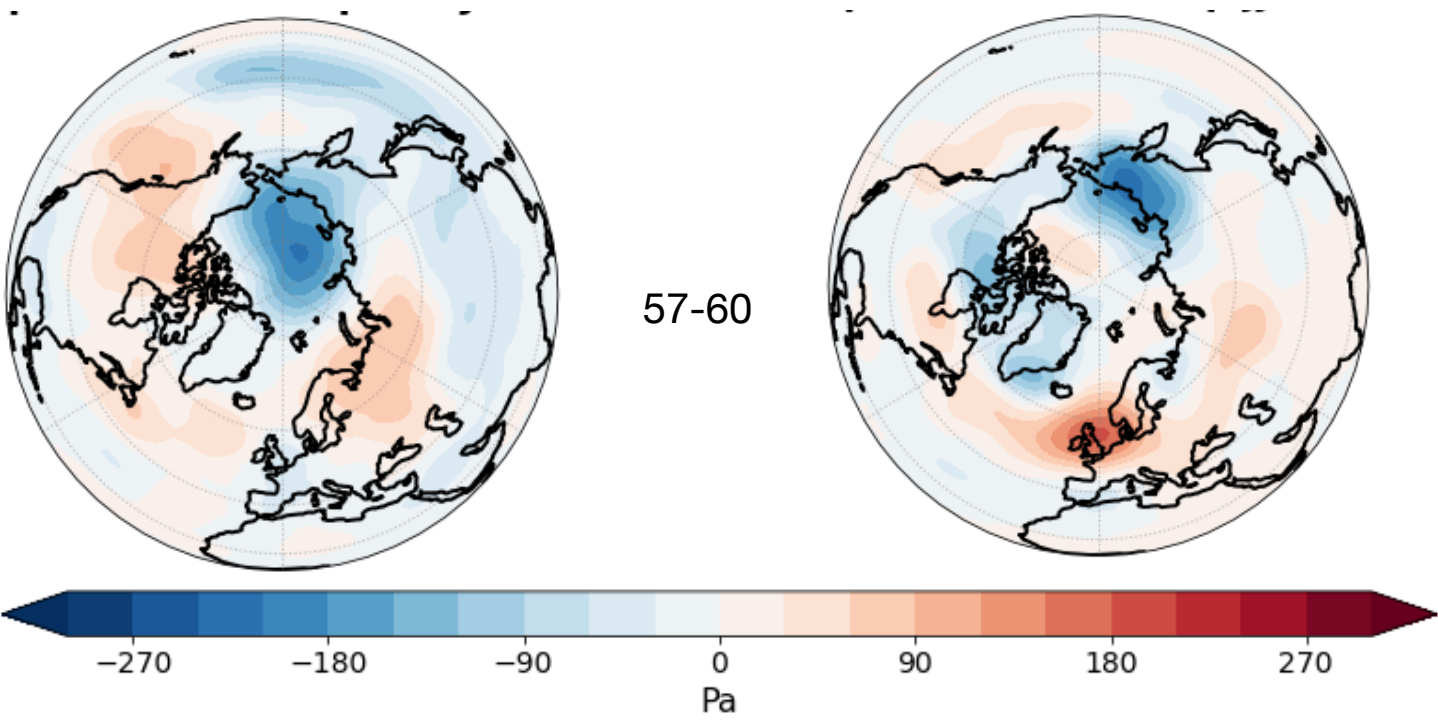

Figure 10. Same as in Figure 10, for DJF. 
https://doi.org/10.5194/wcd-2021-85

Preprint. Discussion started: 4 January 2022

(c) Author(s) 2022. CC BY 4.0 License.

(a)

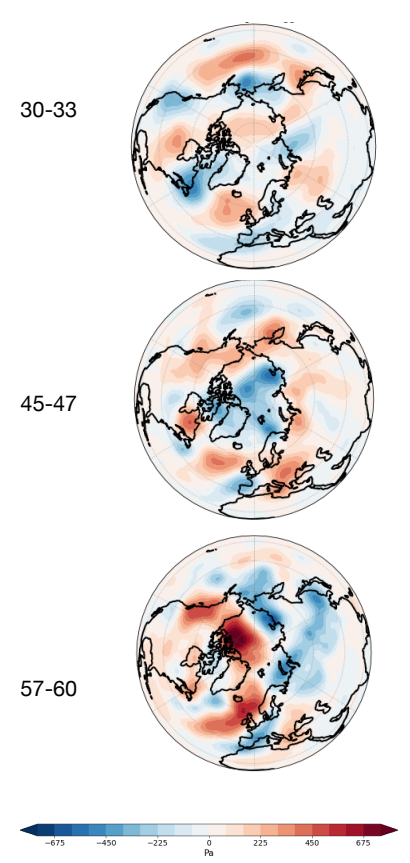

(b)
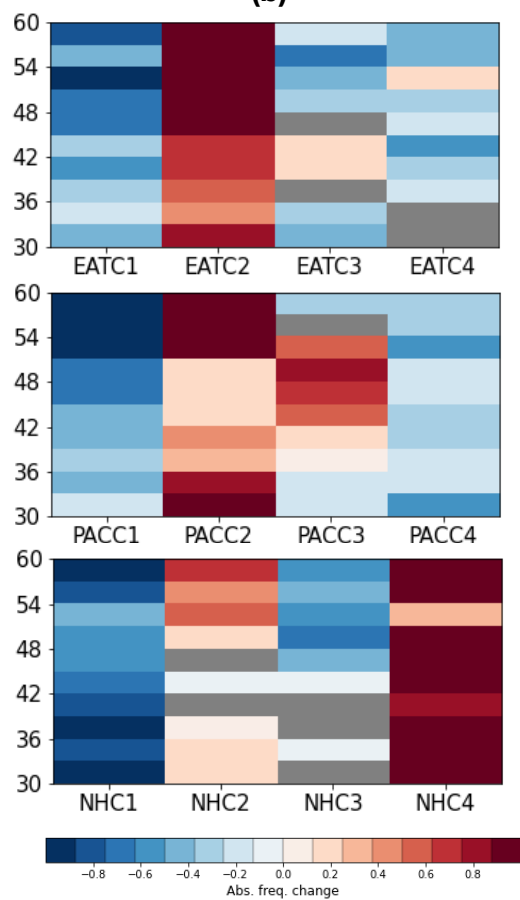

(c)
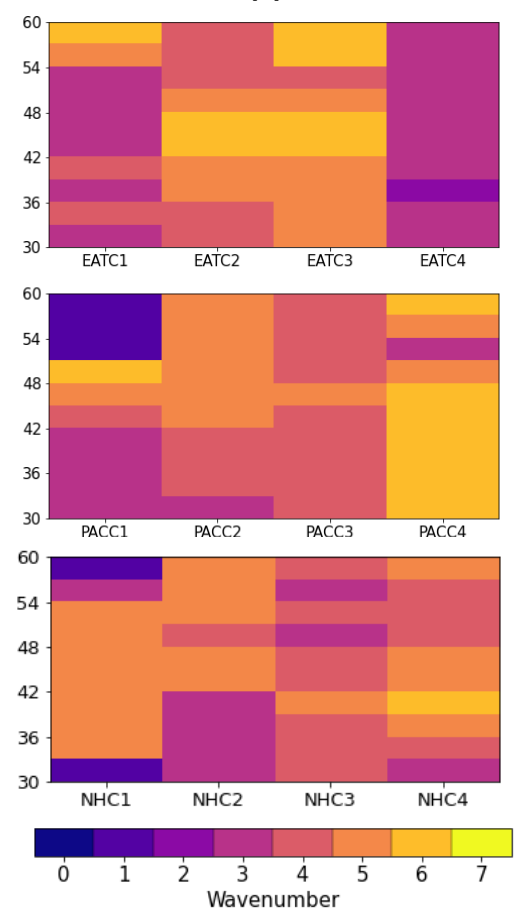

Figure 11. (a) Same as in Figure 9, for 2010 poleward extremes only. The extremes are computed wrt. to 2010 JJA time mean; (b) same as in Figure 6, for 2010 poleward extremes only; (c) same as in Figure 8 for 2010 poleward extremes only. 

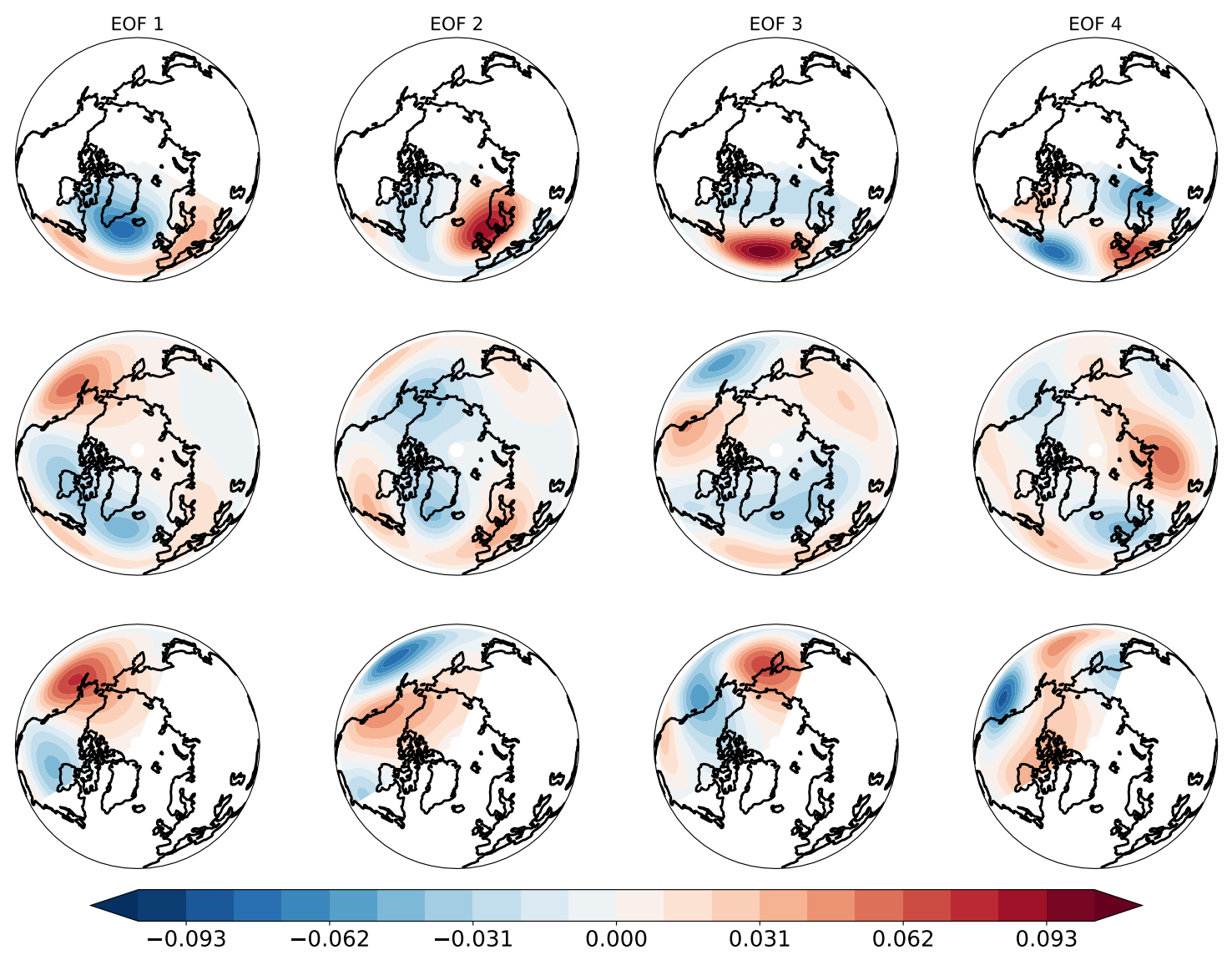

Figure A1. Patterns of the 4 leading EOFs for DJF: EAT (first row), NH (second), PAC (third). 

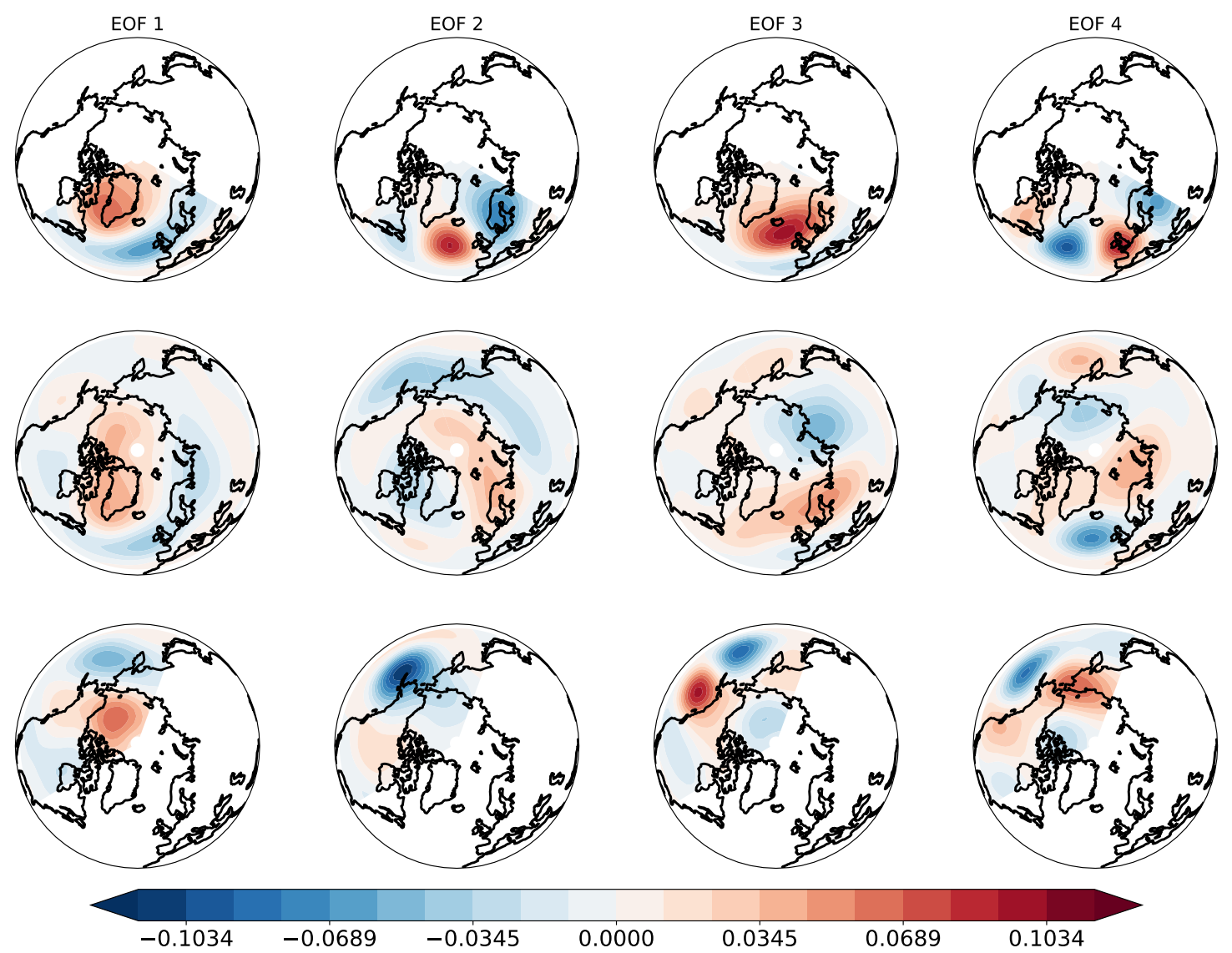

Figure A2. Patterns of the 4 leading EOFs for JJA: EAT (first row), NH (second), PAC (third). 
(a)

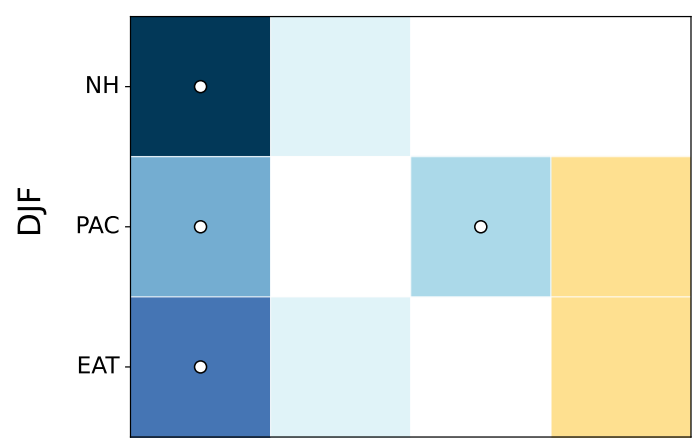

(c)

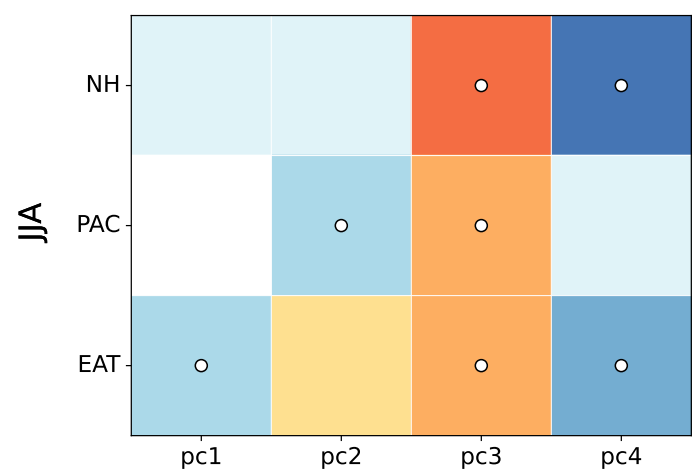

(b)

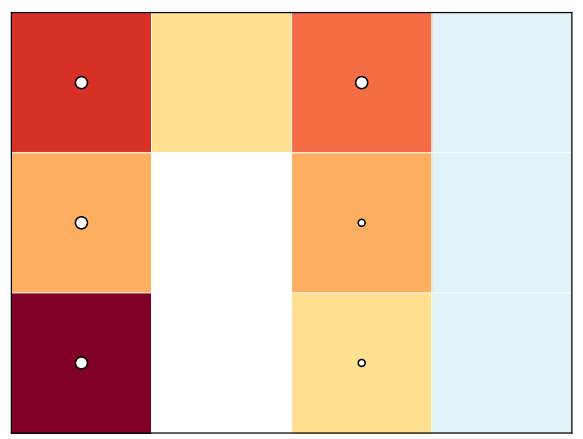

(d)

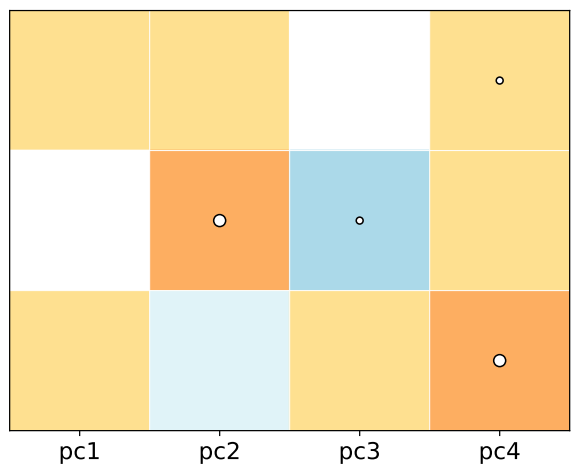

$-0.100-0.075-0.050-0.025 \quad 0.000 \quad 0.025 \quad 0.050 \quad 0.075 \quad 0.100$

Fractional change in PC mean

Figure B1. Fractional change (relative to climatological std. dev.) of the PC mean for the first 4 leading EOFs, as functions of the region of interest, for (a) DJF poleward extremes, (b) DJF equatorward extremes, (c) JJA poleward extremes, (d) JJA equatorward extremes. White dots denote the significance of the change wrt the overall population of events. Large dots denote $99 \%$ significance, small dots denote $95 \%$ significance. 